\title{
A THEORY OF ANALYTIC FUNCTIONS IN BANACH ALGEBRAS
}

\author{
BY \\ E. K. BLUM
}

Introduction. The present paper is concerned with the general problem of extending the classical theory of analytic functions of a complex variable. This question received the attention of Hilbert and F. Riesz, and probably goes back to Volterra. More recently N. Dunford, L. Fantappié, I. Gelfand, E. R. Lorch, A. D. Michal, and A. E. Taylor have contributed to the subject (see bibliography).

Our approach differs from most of the others in two main respects, namely, in the type of domain and range of the functions and in the definition of analyticity. We consider functions which have for their domains and ranges subsets of an abstract commutative Banach algebra with unit and we use a definition of analyticity introduced by E. R. Lorch [1]. It is known [4] that a function analytic by this definition is differentiable in the Fréchet sense but not every Fréchet-differentiable function on a commutative Banach algebra is analytic in the Lorch sense. Accordingly, the Lorch theory is the richer.

For the most part, the development of the primary aspects of the Lorch theory parallels that of the classical theory. Interesting departures occur in the more advanced stages. As one would expect, the Cauchy integral theorem and formula occupy a central position and yield the Taylor expansion in the usual way. With Lorch's work as a foundation, we have extended the theory to include a study of Laurent expansions and analytic continuation. There are also some results on the zeros of polynomials over the algebra, on rational functions and their integrals, and on the singularities of analytic functions. Although the objective of this investigation was essentially analytical, we have also obtained results of an algebraic-topological character (e.g. distribution of singular elements). This was a natural outcome of the algebraic character of the techniques used:

1. Basic concepts. A set, $B$, of elements (denoted by Latin letters $a, b, c, x, y, z, \cdots)$ is a "Banach algebra" if (1) $B$ is an algebra over the complex numbers (denoted by greek letters), (2) $B$ is a complex Banach space, and (3) the norm satisfies the inequality $\|a b\| \leqq\|a\|\|b\|$. In addition, we assume that multiplication is commutative and that $B$ contains a unit element, $e$, with $\|e\|=1$.

We recall without proof some of the salient facts about Banach algebras. Multiplication is continuous in both factors together in the metric topology

Received by the editors March 9, 1953 and, in revised form, January 14, 1954. 
defined by the norm. If $a^{-1}$ exists $\left(a a^{-1}=a^{-1} a=e\right)$, then $a$ is a "regular" element. The set of regular elements will be denoted by $G$. Since $z^{-1}$ is a continuous function of $z, G$ is a topological group relative to multiplication.

$G$ is an open set and is therefore the union of disjoint maximal open connected sets, the components of $G$. The component $G_{1}$ is the maximal connected subgroup of $G$ [4]. If $G$ has a component other than $G_{1}$, then $G$ has infinitely many components [1]. We note that $G_{1}$ contains the complex plane with the origin removed, i.e. the set of elements of the form $\lambda e, \lambda \neq 0$.

An element which is not regular is "singular." The set of singular elements is closed and contains at least the zero element, 0 . If it contains only 0 , then $B$ is isomorphic to the field of complex numbers [8].

If $I \subset B$ is a closed proper ideal $(I \neq B)$, then the quotient algebra $B / I$ is also a commutative Banach algebra with unit [3]. If $M$ is a maximal ideal, then $B / M$ is isomorphic to the field of complex numbers. The natural homomorphism $B \rightarrow B / M$ induces a homomorphism of the algebra onto the complex numbers. If $x \in B$, we let $x(M)$ denote the complex number onto which $x$ is mapped [3]. If $x$ is fixed and $M$ varies over the set of maximal ideals, $\mathfrak{M}$, then $x(M)$ may be regarded as a complex-valued function on $\mathfrak{M}$. Gelfand shows that this function has the following properties: (1) If $x=x_{1}+x_{2}$, then $x(M)=x_{1}(M)+x_{2}(M)$. (2) If $x=x_{1} \cdot x_{2}$, then $x(M)=x_{1}(M) \cdot x_{2}(M)$. If $\lambda$ is complex, then $\lambda \cdot x(M)=(\lambda x)(M)$. (3) $e(M)=1$. (4) $|x(M)| \leqq\|x\|$. (5) If $M \neq M^{\prime}$, there is an $x$ in $B$ such that $x(M) \neq x\left(M^{\prime}\right)$. (6) If $x(M)$ is not zero for any $M$ in $\mathfrak{M}$, then there is a $y$ in $B$ such that $y(M)=1 / x(M)$.

An element $b$ is "nilpotent" if $b^{n}=0$ for some positive integer $n$. It is "quasi-nilpotent" if for all complex $\lambda, \lim _{n \rightarrow \infty}(\lambda b)^{n}=0$. [1]. The set of quasinilpotents is called the "radical" of $B$. An element $b$ is quasi-nilpotent if and only if $\lim _{n \rightarrow \infty}\left(\left\|b^{n}\right\|\right)^{1 / n}=0$. For every $x$ in $B, \lim _{n \rightarrow \infty}\left(\left\|x^{n}\right\|\right)^{1 / n}=\max \{|x(M)|\}$, $M$ in $\mathfrak{M}$. Thus the radical is the intersection of all the maximal ideals of $B$. $\mathfrak{M}$ may be topologised by defining a neighborhood of $M_{0}$ in $\mathfrak{M}$ as the set of all $M$ in $\mathfrak{M}$ such that $\left|x_{i}(M)-x_{i}\left(M_{0}\right)\right|<\epsilon$ where the $x_{i}, i=1,2, \cdots, n$, are any finite number of arbitrary elements in $B$ and $\epsilon>0$ is any real number. With this topology, $\mathfrak{M}$ is a compact Hausdorff space and the functions $x(M)$ are continuous on $\mathfrak{M}$.

The algebra $B$ is "reducible" if it is the direct sum of proper ideals which are also $B$-algebras with units, that is, $B=B_{1}+B_{2}$, where $B_{i} \neq\{0\}$. Reducibility is equivalent to the existence of an idempotent element $e_{j}\left(e_{j}^{2}=e_{j}\right)$ such that $e_{j} \neq e, e_{j} \neq 0$. A notion closely related to reducibility is that of the spectrum of an element. The "spectrum" of $b$ is the set of complex numbers $\lambda$ such that $(b-\lambda e)$ is singular. The number $\lambda$ is in the spectrum of $b$ if and only if there is an $M$ in $\mathfrak{M}$ such that $b(M)=\lambda$. The algebra $B$ is irreducible if and only if the spectrum of every element is a connected set in the complex plane. An element $b$ is regular if and only if $b(M) \neq 0$ for every $M$ in $\mathfrak{M}$.

2. Analytic functions. All functions in $\$ \S 2-4$ are assumed to be single- 
valued. The concepts "curve," "rectifiable," "length," "integration on a curve" are defined in the natural way [1]. The classical theorems on Riemann integration are valid since the proofs require only those properties of the complex numbers which are also possessed by the algebra $B$, the norm in $B$ playing the role of the modulus.

We now state the definition of analyticity, given by Lorch [1], which shall be used herein.

Definition 2.1. Let $z_{0}$ be a point in $B$ and let $N\left(z_{0}\right)$ be a neighborhood of $z_{0}$. Let $f(z)$ be a function defined on $N\left(z_{0}\right)$ with range in $B . f(z)$ is "differentiable" at $z_{0}$ if there is an element, $f^{\prime}\left(z_{0}\right)$, in $B$ with the property that for any real $\epsilon>0$ there is a $\delta>0$ such that for all $z$ in $B$ with $\left\|z-z_{0}\right\|<\delta$

$$
\left\|f(z)-f^{\prime}\left(z_{0}\right)-\left(z-z_{0}\right) f^{\prime}\left(z_{0}\right)\right\| \leqq \epsilon\left\|z-z_{0}\right\| .
$$

The element $f^{\prime}\left(z_{0}\right)$ is the "derivative" of $f(z)$ at $z_{0}$. The function $f(z)$ is "analytic at $z_{0}$ " if it has a derivative at all points in some neighborhood of $z_{0}$.

3. The Cauchy theorem and formula. The following theorem was stated by Lorch [1]:

THEOREM 3.1. Let $E$ be a convex region of $B$. If $f(z)$ is analytic in $E$ and $K$ is an arbitrary rectifiable closed curve in $E$, then $\int_{K} f(z) d z=0$.

The convexity of $E$ permits the construction of a proof along classical lines in which the theorem is established first for a triangle, then a polygon, and finally for the curve $K$. The proof for the triangle is based on its compactness.

We now prove the Cauchy integral theorem for a Banach algebra.

THEOREM 3.2. Let $f(z)$ be analytic in a region $E$. Let $K$ be an arbitrary rectifiable closed curve which is homotopic to a point in $E$. Then $\int_{K} f(z) d z=0$.

(REMARK. When dealing with homotopy in an open set, there is no loss of generality in assuming that all the curves are rectifiable. For example, in the proof which follows we can assume that the curves $K_{i}, i=1, \cdots, m$, are rectifiable since each $K_{i}$ can be replaced by the homotopic polygonal curve consisting of the line segments joining the points $z_{0}, z_{i 1}, z_{i 2}, \cdots, z_{i, n-1}, z_{n}$ on $K_{i}$.)

Proof. Let $K$ be parametrized by $z=\phi(t)$, where $\phi(0)=\phi(1)=z_{0}$. Since $K$ is homotopic to $z_{0}$, there is a function of two real variables, $F(s, t)$, continuous on the unit square $0 \leqq s \leqq 1,0 \leqq t \leqq 1$, in both variables together and such that $F(0, t)=\phi(t), F(1, t) \equiv z_{0}, F(s, 0)=F(s, 1)=z_{0}$, and $F(s, t)$ is in $E$ for every $(s, t)$ in the unit square.

Since the mapping $F$ is continuous on the compact unit square, its image, $\{F(s, t)\}$, is a compact set in $E$. Hence, there is a minimum distance, $\rho>0$, from this image set to the boundary of $E$.

Now, $F$ is uniformly continuous. Hence, there is a $\delta>0$ such that $\| F\left(s^{\prime}, t^{\prime}\right)$ 
$-F(s, t) \|<\rho / 2$ for all pairs $(s, t),\left(s^{\prime}, t^{\prime}\right)$ with $\left|s^{\prime}-s\right|<\delta$ and $\left|t^{\prime}-t\right|<\delta$. We choose $s_{1}$ such that $\left|s_{1}\right|<\delta$ and $0=t_{0}<t_{1}<\cdots<t_{n}=1$ such that $\left|t_{i}-t_{i-1}\right|$ $<\delta$. Let $S\left(z_{0}\right)$ be the sphere $\left\|z-z_{0}\right\|<\rho$. Let $z_{0 i}=F\left(0, t_{i}\right), i>0$, and $z_{1 i}=F\left(s_{1}, t_{i}\right)$ and let $K_{1}$ be the curve defined by $F\left(s_{1}, t\right), 0 \leqq t \leqq 1$. Now, $\left\|z_{11}-z_{0}\right\|<\rho / 2$, $\left\|z_{12}-z_{11}\right\|<\rho / 2,\left\|z_{02}-z_{01}\right\|<\rho / 2$, and $\left\|z_{01}-z_{0}\right\|<\rho / 2$. Therefore, for $\left|t-t_{1}\right|<\delta$ we have $\left\|F\left(s_{1}, t\right)-z_{0}\right\| \leqq\left\|F\left(s_{1}, t\right)-z_{11}\right\|+\left\|z_{11}-z_{0}\right\|<\rho,\left\|F(0, t)-z_{0}\right\| \leqq \| F(0, t)$ $-z_{01}\|+\| z_{01}-z_{0} \|<\rho$. Thus, these points are in $S\left(z_{0}\right)$, which is a convex set contained in $E$. Hence, the path consisting of the arc of $K$ joining $z_{0}$ to $z_{01}$, the arc of $K_{1}$ joining $z_{0}$ to $z_{11}$, and the line segment joining $z_{01}$ to $z_{11}$ (denoted by $\left.L_{1}\right)$ is contained in $S\left(z_{0}\right)$. By Theorem 3.1 we have

$$
\int_{z_{0}, K}^{z_{01}} f(x) d x+\int_{z_{01}, L_{1}}^{z_{11}} f(x) d x=\int_{z_{0}, K_{1}}^{z_{11}} f(x) d x .
$$

Next we let $S\left(z_{01}\right)$ be the sphere $\left\|z-z_{01}\right\|<\rho$. As above, the arc of $K$ from $z_{01}$ to $z_{02}$, the line segment $L_{2}$ joining $z_{02}$ to $z_{12}$, the arc of $K_{1}$ from $z_{12}$ to $z_{11}$, and the line segment $L_{1}$ are all in $S\left(z_{01}\right)$. By Theorem 3.1 again,

$$
-\int_{z_{01}, L_{1}}^{z_{11}} f(x) d x+\int_{z_{01}, K}^{z_{02}} f(x) d x+\int_{z_{02}, L_{2}}^{z_{12}} f(x) d x=\int_{z_{11}, K_{1}}^{z_{12}} f(x) d x .
$$

Proceeding in this way at each of the points $z_{0 i}, i=0,1, \cdots, n-1$, we obtain a set of $n$ equations of the above type. Adding these equations, we see that all integrals over the line segments cancel, leaving

$$
\int_{K} f(x) d x=\int_{K_{1}} f(x) d x .
$$

Now consider the curve $K_{2}: F\left(s_{2}, t\right)$ where $\left|s_{2}-s_{1}\right|<\delta$. Applying the above procedure, we obtain $\int_{K_{2}} f(x) d x=\int_{K_{1}} f(x) d x$. After a finite number of steps, we obtain $\int_{K_{m}} f(x) d x=\int_{K} f(x) d x$, where $K_{m}$ is the "null" curve, $F(1, t) \equiv z_{0}$. Thus $\int_{K_{m}} f(x) d x=0$ and the theorem is proven.

Definition 3.1. The set of points of the form $z+\lambda e$ where $z$ is a fixed element in $B$ and $\lambda$ varies over the complex numbers is the " $z$-plane $\left({ }^{1}\right) . "$

Definition 3.2. Let $K:\{z+\lambda(t) e\}$ be a simple closed curve in the $z$-plane (i.e. $\lambda(t)$ is continuous and complex, $0 \leqq t \leqq 1)$. A point $z+\lambda e$ in the $z$-plane is "interior to $K$ " if the complex number $\lambda$ is interior to the simple closed curve, $\zeta=\lambda(t)$, in the complex plane.

An analogue of the Cauchy formula was stated for a neighborhood by Lorch. The following theorem is a somewhat sharper formulation but is not quite the complete analogue (see $\$ 4$ ).

THEOREM 3.3. Let $f(z)$ be analytic in a region $E \subset B$. Let $z_{0}$ be a point in $E$

(1) More generally we may consider the set $\{z+\lambda g\}$ where $g$ is a regular element. We shall call this set the $(z+\lambda g)$-plane. 
and $S\left(z_{0}\right)$ the sphere of maximum radius, $\rho$, with center $z_{0}$ which is contained in $E$. If $K$ is the curve $\left\{z_{0}+(\mu \exp i \theta) g \mid 0 \leqq \theta \leqq 2 \pi, g \in G, \mu\|g\|=\rho / 2\right\}$, and $S\left(z_{0}, \delta\right)$ is the sphere with center $z_{0}$ and radius $\delta=\mu /\left(4\left\|g^{-1}\right\|\right)$, then for all $z$ in $S\left(z_{0}, \delta\right)$

$$
f(z)=\frac{1}{2 \pi i} \int_{K}(x-z)^{-1} f(x) d x .
$$

Proof. For all points $x$ on $K$ we have $\left\|x-z_{0}\right\|=|\mu|\|g\|$. If $z$ is in $S\left(z_{0}, \delta\right)$, then $z=z_{0}+b$ where $\|b\|<\mu /\left(4\left\|g^{-1}\right\|\right) \leqq \mu\|g\| / 4$. Thus, $|(x-z)(M)|$ $=\left|\left(x-z_{0}-b\right)(M)\right| \geqq\left|\left(x-z_{0}\right)(M)\right|-|b(M)| \geqq|\mu||g(M)|-\mu / 4|| g^{-1} \|>0$, for every maximal ideal $M$. This implies that $(x-z)^{-1}$ exists for all $x$ in $K$. Since $K \subset S\left(z_{0}\right)$, the integrand is defined.

Now let $K^{\prime}$ be the curve $\{z+(\mu \exp i \theta) g\}$ and let $\bar{K}^{\prime}$ be the set $\{z+\lambda g|| \lambda \mid \leqq \mu\}$. Clearly $\bar{K}^{\prime} \subset S\left(z_{0}\right)$. Thus $f(z)$ is analytic in $\bar{K}^{\prime}$ and since the Cauchy formula holds in the $(z+\lambda g)$ plane,

$$
f(z)=\frac{1}{2 \pi i} \int_{K^{\prime}}(x-z)^{-1} f(x) d x .
$$

If $x$ is in $K$, the sphere $S\left(x, \mu / 2\left\|g^{-1}\right\|\right)$ is contained in $S\left(z_{0}\right)$. Let $y$ be in $S\left(x, \mu / 2\left\|g^{-1}\right\|\right)$. Then $y=x+c$ where $\|c\|<\mu / 2\left\|g^{-1}\right\|$. Hence, for every maximal ideal $M$,

$$
|(y-z)(M)| \geqq|(x-z)(M)|-|c(M)| \geqq 3 \mu / 4\left\|g^{-1}\right\|-\mu / 2\left\|g^{-1}\right\|>0
$$

so that $(y-z)^{-1} f(y)$ is defined and analytic in $y$ in the sphere $S\left(x, \mu / 2\left\|g^{-1}\right\|\right)$.

Now choose $0=\theta_{1}<\theta_{2}<\cdots<\theta_{n}=2 \pi$ such that for the corresponding points $x_{1}, x_{2}, \cdots, x_{n}=x_{1}$ on $K$ the arc length from $x_{j}$ to $x_{j+1}$ is less than $\mu / 4\left\|g^{-1}\right\|, j=1, \cdots, n-1$. For $x_{j}=z_{0}+\left(\mu \exp i \theta_{j}\right) g$, choose $x_{j}^{\prime}=z+\left(\mu \exp i \theta_{j}\right) g$ on $K^{\prime}$. Let $L_{j}$ denote the line segment joining $x_{j}$ to $x_{j}^{\prime}$. Clearly, $L_{j} \subset S\left(x_{j}\right.$, $\left.\mu / 2\left\|g^{-1}\right\|\right)$ so that $L_{j} \subset S\left(z_{0}\right)$ and $(x-z)^{-1}$ exists for all $x$ on $L_{j}$.

Finally, let $C_{j}$ be the closed curve consisting of the arc of $K$ from $x_{j}$ to $x_{j+1}$, followed by the line segment $L_{j+1}$, then by the arc of $K^{\prime}$ from $x_{j+1}^{\prime}$ to $x_{j}^{\prime}$ and lastly the line segment $L_{j}$. The preceding remarks show that $C_{j} \subset S\left(x_{j}\right.$, $\left.\mu / 2\left\|g^{-1}\right\|\right)$. Since the function $g(x) \equiv(1 / 2 \pi i)(x-z)^{-1} f(x)$ is analytic for $x$ in $S\left(x_{j}, \mu / 2\left\|g^{-1}\right\|\right)$, the Cauchy integral theorem yields the result

$$
\begin{aligned}
\int_{C_{j}} g(x) d x= & \int_{x_{j}, K}^{x_{j+1}} g(x) d x+\int_{x_{j+1}, L_{j+1}}^{x_{j+1}} g(x) d x+\int_{x_{j^{\prime}+1}, K^{\prime}}^{x_{j^{\prime}}} g(x) d x \\
& +\int_{x_{i^{\prime}, L i}}^{x_{j}} g(x) d x=0 .
\end{aligned}
$$

Adding these equations for $j=1, \cdots, n-1$, we obtain

$$
\frac{1}{2 \pi i} \int_{K}(x-z)^{-1} f(x) d x=\frac{1}{2 \pi i} \int_{K^{\prime}}(x-z)^{-1} f(x) d x .
$$


But the right-hand member equals $f(z)$. This establishes the theorem.

The formula for the derivative is obtained in similar fashion and for the higher derivatives by induction as in the classical theory.

\section{Taylor expansions.}

Definition 4.1. A series $P\left(z \mid z_{0}\right)=\sum_{n=0}^{\infty} b_{n}\left(z-z_{0}\right)^{n}$ where the $b_{n}$ are elements of $B$ is a "Taylor series" or "power series" around $z_{0}$. It is "superabsolutely" convergent (s.-a. convergent) if the series $\sum_{0}^{\infty}\left\|b_{n}\right\|\left\|z-z_{0}\right\|^{n}$ converges. It is "absolutely" convergent if $\sum_{0}^{\infty}\left\|b_{n}\left(z-z_{0}\right)^{n}\right\|$ converges.

Definition 4.2. The "radius of convergence" of the series $P\left(z \mid z_{0}\right)$ is defined as $1 / \lim \sup _{n \rightarrow \infty}\left(\left\|b^{n}\right\|\right)^{1 / n}$.

The classical theorems on power series are easily established. However, Taylor's theorem must be proven in two steps. First, it is shown that the Taylor series of $f(z)$ equals $f(z)$ in a neighborhood of the center of expansion, $z_{0}$. Then the identity theorem is proven. (Here, it is important to note that we require two analytic functions to be equal throughout a neighborhood of $z_{0}$ in order to prove them identical. The classical condition that they be equal at a sequence of points approaching $z_{0}$ is known to be insufficient.) Combining these two results, we obtain equality of $f(z)$ and its power series in the largest sphere contained in the region of analyticity.

This, in turn, yields the complete analogue of the Cauchy formula. With reference to Theorem 3.3 , we state it as follows.

TheOREM 4.1. Let $K$ be the curve $\left\{z_{0}+(\mu \exp i \theta) e \mid 0 \leqq \theta \leqq 2 \pi, \mu<\rho\right\}$. Then formula (1) of Theorem 3.3 holds for all $z$ such that $\left\|z-z_{0}\right\|<\mu$.

5. Analytic continuation. The concepts of analytic continuation, functional element, and complete analytic function are taken over directly from the classical theory. We shall be especially concerned with the singularities of an analytic function. For the precise definitions, the reader is referred to Courant's Funktionentheorie, p. $376 \mathrm{ff}$. The following example will illustrate how the definitions are used here.

Consider the function defined by the formula $z^{-1}$. In each component of $G, z^{-1}$ is a distinct single-valued analytic function. Consider the principal component, $G_{1} . G_{1}$ is the domain of a complete analytic function defined by $z^{-1}$, for suppose $d$ is a boundary point of $G_{1}$. Let $K$ be a path joining $e$ to $d$ such that $K$ is in $G_{1}$ except for the end point $d$. Suppose further that it is possible to continue a functional element $P_{1}(z \mid e)$, at $e$, along $K$ so as to obtain a functional element $P_{n}(z \mid d)$ at $d$. We assume, as we may, that the functional elements $P_{1}(z \mid e), P_{2}\left(z \mid z_{2}\right), \cdots, P_{n-1}\left(z, z_{n-1}\right), P_{n}(z, d)$ are power series, the center of each being in the sphere of s.-a. convergence of the preceding. Now, there is a sequence of regular elements $\left\{g_{j}\right\}$ on $K$ such that $\lim _{j} g_{j}=d$ and $g_{j}$ is in the sphere of s.-a. convergence of $P_{n-1}\left(z \mid z_{n-1}\right)$. Then we have $P_{n-1}\left(g_{j} \mid z_{n-1}\right)=g_{j}^{-1}$ and $e=\lim _{j}\left[g_{j} \cdot P_{n-1}\left(g_{j} \mid z_{n-1}\right)\right]=d \cdot P_{n-1}\left(d \mid z_{n-1}\right)$. This implies $d$ is in $G$, which is a contradiction. Thus $d$ is a " $K$-singularity" of $z^{-1}$ and $z^{-1}$ cannot be continued outside of $G$. 
6. The Laurent expansion. A Laurent expansion about a point $z_{0}$ is a series of the form $\sum_{-\infty}^{\infty} b_{n}\left(z-z_{0}\right)^{n}, b_{n}$ in $B$, defined for those $z$ such that $\left(z-z_{0}\right)^{-1}$ exists and the two series $\sum_{0}^{\infty} b_{n}\left(z-z_{0}\right)^{n}$ and $\sum_{-1}^{-\infty} b_{n}\left(z-z_{0}\right)^{n}$ are convergent. A Laurent expansion may be defined in several disjoint regions in each of which it may define a different analytic function; e.g. $z^{-1}$. With this possibility in mind, we prove the main theorem.

TheOREM 6.1. Let $g$ be an arbitrary element in $G$, and let $E$ denote the set $\left\{z_{0}+\lambda g\left|0 \leqq r_{1}<\right| \lambda \mid<r_{2}\right\}$. Setting $\mu_{\lambda}=\min \left\{r_{2}-|\lambda|,|\lambda|-r_{1}\right\}$, we write $S(\lambda)$ for the sphere $\left\|z-\left(z_{0}+\lambda g\right)\right\|<\mu_{\lambda} /\left\|g^{-1}\right\|$ and $U=U_{r_{1}<|\lambda|<r_{2}} S(\lambda)$. If $D$ is a region such that $E \subset D \subset U$ and $f(z)$ is a function which is single-valued and analytic in $D$, then for each $z$ in $U$ there are two curves $K_{j}(z):\left\{z_{0}+\left(\rho_{j} \exp i t\right) g\right.$, $0 \leqq t \leqq 2 \pi\}, j=1,2$, with $r_{1}<\rho_{1}<\rho_{2}<r_{2}$ such that:

(1) $\phi(z)=\frac{1}{2 \pi i} \int_{K_{2}(z)}(x-z)^{-1} f(x) d x-\frac{1}{2 \pi i} \int_{K_{1}(z)}(x-z)^{-1} f(x) d x$ is a well-defined single-valued analytic function of $z$;

(2) for all $z$ in $U, \phi(z)=\sum_{-\infty}^{\infty} a_{n}\left(z-z_{0}\right)^{n}$ where

$$
a_{n}=\frac{1}{2 \pi i} \int_{K}\left(x-z_{0}\right)^{-n-1} f(x) d x
$$

and $K$ is any curve of the form $\left\{z_{0}+(r \exp i t) g \mid r_{1}<r<r_{2}, 0 \leqq t \leqq 2 \pi\right\}$;

(3) for all $z$ in $D, \phi(z)=f(z)$.

Proof. Let $z$ be in $U . z=z_{0}+\lambda g+h$ where $\|h\|=\mu_{\lambda}-\epsilon /\left\|g^{-1}\right\|, \epsilon>0$. For all $M$ in $\mathfrak{M},|g(M)|=1 /\left|g^{-1}(M)\right| \geqq 1 /\left\|g^{-1}\right\|$. Hence, $|h(M)| \leqq\|h\|<\mu_{\lambda} /\left\|g^{-1}\right\|$ $\leqq|g(M)| \cdot \min \left\{r_{2}-|\lambda|,|\lambda|-r_{1}\right\}$, or $|h(M)| \leqq|g(M)| \cdot\left(r_{2}-\epsilon-|\lambda|\right)$ and $|h(M)| \leqq|g(M)|\left(|\lambda|-r_{1}-\epsilon\right)$. Then we have $\left|\left(z-z_{0}\right)(M)\right|=|\lambda g(M)+h(M)|$ $\leqq|\lambda| \cdot|g(M)|+|h(M)| \leqq|\lambda| \cdot|g(M)|+|g(M)|\left(r_{2}-\epsilon-|\lambda|\right)=\left(r_{2}-\epsilon\right)|g(M)|$. Also $\left|\left(z-z_{0}\right)(M)\right| \geqq|\lambda| \cdot|g(M)|-|h(M)| \geqq|\lambda||g(M)|-|\lambda||g(M)|$ $+\left(r_{1}+\epsilon\right)|g(M)|=\left(r_{1}+\epsilon\right)|g(M)|$.

Now let $\rho_{1}=r_{1}+\delta, \rho_{2}=r_{2}-\delta$ where $0<\delta<\epsilon$, and consider the curves $K_{j}(z)=\left\{z_{0}+\left(\rho_{j} \exp i t\right) g\right\}, j=1,2$. For all $x$ on $K_{2}(z)$ we have $|(x-z)(M)|$ $\geqq \||g(M)| \rho_{2}-\left|\left(z_{0}-z\right)(M)\right| \geqq|g(M)|\left(r_{2}-\delta\right)-\left(r_{2}-\epsilon\right)|g(M)|=(\epsilon-\delta)|g(M)|$ $>0$. Likewise for $x$ on $K_{1}(z),|(x-z)(M)| \geqq|g(M)|\left(r_{1}+\epsilon\right)-|g(M)|\left(r_{1}+\delta\right)$ $=|g(M)|(\epsilon-\delta)>0$. Thus the integrand in both of the integrals of (1) is defined. Furthermore, although the choice of the curves $K_{j}(z)$ is not uniquely determined by $z$, any pair of curves which satisfy the specified conditions will yield the same value for each of the integrals in (1). This is an immediate consequence of the Cauchy theorem. Hence, the value of $\phi(z)$ is uniquely determined by $z$.

Now, for $x$ on $K_{2}(z)$ we have $\left(x-z_{0}\right)^{-1}=\left(1 / \rho_{2}\right) g^{-1} \exp (-i t)$. Hence, $\left(x-z_{0}\right)^{-1}\left(z-z_{0}\right)=\left(1 / \rho_{2}\right) g^{-1}(\lambda g+h) \exp (-i t)=\left(1 / \rho_{2}\right)\left(\lambda+h g^{-1}\right) \exp (-i t)$ and $\left\|\left(x-z_{0}\right)^{-1}\left(z-z_{0}\right)\right\| \leqq\left(1 / \rho_{2}\right)\left[|\lambda|+\|h\|\left\|g^{-1}\right\|\right] \leqq\left(1 / \rho_{2}\right)\left[|\lambda|+\left(r_{2}-|\lambda|-\epsilon\right)\right.$ 
$\left.\cdot\left(1 /\left\|g^{-1}\right\|\right)\left\|g^{-1}\right\|\right]=\left(r_{2}-\epsilon\right) / \rho_{2}=\left(r_{2}-\epsilon\right) /\left(r_{2}-\delta\right)<1$. The last fraction is less than 1. Therefore, we may write $(x-z)^{-1}=\left(x-z_{0}\right)^{-1}\left\{e+\sum_{n=1}^{\infty}\left[\left(z-z_{0}\right)\right.\right.$ $\left.\left.\left(x-z_{0}\right)^{-1}\right]^{n}\right\}$ since the series converges. The convergence is absolute and uniform in $x$ for $x$ on $K_{2}(z)$.

For $x$ on $K_{1}(z),\left(x-z_{0}\right)=\left(\rho_{1} \exp i t\right) g$. Since $\left(z-z_{0}\right)^{-1}=(\lambda g+h)^{-1}=(1 / \lambda) g^{-1}$ $\left(e+h g^{-1} / \lambda\right)^{-1}$, we have

$$
\left(x-z_{0}\right)\left(z-z_{0}\right)^{-1}=\left(\rho_{1} \exp (i t) / \lambda\right)\left[e-h g^{-1} / \lambda+\left(h g^{-1} / \lambda\right)^{2}-\cdots\right],
$$

the series being super-absolutely convergent because $\left\|h g^{-1}\right\| /|\lambda| \leqq\|h\|$ . $\left\|g^{-1}\right\| /|\lambda|=1 /|\lambda|\left(\mu_{\lambda}-\epsilon /\left\|g^{-1}\right\|\right)\left\|g^{-1}\right\|=\left(\mu_{\lambda}-\epsilon\right) /|\lambda|<1$. In fact, $\|\left(x-z_{0}\right)$ $\cdot\left(z-z_{0}\right)^{-1}|| \leqq\left(\rho_{1} /|\lambda|\right)\left[1-\left(\mu_{\lambda}-\epsilon\right) /|\lambda|\right]^{-1}=\rho_{1} /\left(|\lambda|-\mu_{\lambda}+\epsilon\right) \leqq \rho_{1} /\left(r_{1}+\epsilon\right)<1$. Thus we may write $(x-z)^{-1}=-\left(z-z_{0}\right)^{-1}\left\{e+\sum_{n=1}^{\infty}\left[\left(z-z_{0}\right)^{-1}\left(x-z_{0}\right)\right]^{n}\right\}$. Again the series converges uniformly in $x$ for $x$ on $K_{1}(z)$. Term-by-term integration yields the result (2) of the theorem if we observe that $a_{n}=(1 / 2 \pi i)$ $\cdot \int_{K j(z)}\left(x-z_{0}\right)^{-n-1} f(x) d x$ where $j=2$ for $n \geqq 0$ and $j=1$ for $n<0$, and by the Cauchy theorem $\int_{K j(z)}\left(x-z_{0}\right)^{-n-1} f(x) d x=\int_{K}\left(x-z_{0}\right)^{-n-1} f(x) d x$ where $K$ is any curve of the form $\left\{z_{0}+(r \exp i t) g \mid r_{1}<r<r_{2}, 0 \leqq t \leqq 2 \pi\right\}$.

For $n \geqq 0$,

$$
\begin{aligned}
a_{n} & =\frac{1}{2 \pi i} \int_{K}\left[f(x) /(r \exp i t)^{n+1}\right]\left(g^{-1}\right)^{n+1} d x \\
& =\frac{g^{-n-1}}{2 \pi} \int_{0}^{2 \pi}\left[f(x) /(r \exp i t)^{n+1}\right] g \cdot r \exp (i t) d t
\end{aligned}
$$

so that $\left\|a_{n}\right\| \leqq(1 / 2 \pi)\left(\left\|g^{-1}\right\| n\left\|g^{-1} g\right\| \cdot m / r^{n}\right) 2 \pi=\left\|g^{-1}\right\| n \cdot m / r^{n}$, where $\quad m$ $=\max \{\|f(x)\|, x$ on $K\}$. Thus the series of positive powers of the Laurent series in (2) must be s.-a. convergent for $\left\|z-z_{0}\right\|_{0}<r /\left|g^{-1}\right|<r_{2} /\left|g^{-1}\right|$, or since $r$ may be taken arbitrarily close to $r_{2}$, for $\left\|z-z_{0}\right\|<r_{2} /\left\|g^{-1}\right\| \leqq r_{2}\|g\|$.

For $n<0,\left\|a_{n}\right\| \leqq(1 / 2 \pi) m r^{-n}\|g\|^{-n} \cdot 2 \pi$, where $m=\max \{\|f(x)\| x$ on $K\}$. Thus the series of negative powers converges super-absolutely for $\left\|\left(z-z_{0}\right)^{-1}\right\|$ $<1 /\left(r_{1}\|g\|\right)$. For $z$ in $U,\left\|\left(z-z_{0}\right)^{-1}\right\| \leqq\left\|g^{-1}\right\| / r_{1}$ (by fourth paragraph of this proof). Since $\left\|g^{-1}\right\| / r_{1} \geqq 1 /\left(r_{1}\|g\|\right)$, the series of negative powers may not be s.-a. convergent for all $z$ in $U$, although it converges for these $z$. If $g=e$, then $\left\|g^{-1}\right\|=\|g\|=1$ and this difficulty does not arise. Likewise, if $r_{1}=0$, the series of negative powers is absolutely convergent and s.-a. convergent.

Now suppose $z$ is in $D$. As above, $z=z_{0}+\lambda g+h$ where $\|h\|=\left(\mu_{\lambda}-\epsilon\right) /\left\|g^{-1}\right\|$. Choose $K_{j}(z), j=1,2$, as described in the proof of (1) and choose $K_{\lambda}$ as the curve $\left\{z_{0}+\lambda g+(r \exp i t) g, 0 \leqq t \leqq 2 \pi\right\}$ where $r=\mu_{\lambda}-\delta, 0<\delta<\epsilon$. Consider the set $F=\left\{z_{0}+\zeta g\left|r_{1}<\right| \zeta\left|<r_{2},\right| \zeta-\lambda \mid>\mu_{\lambda}-\epsilon\right\}, F \subset E$ and $K_{\lambda} \subset F$ and $K_{j}(z) \subset F$. We have for all $M$ in $\mathfrak{M}$ and $x$ in $F|(x-z)(M)|=\left|\left(z_{0}+\zeta g-z\right)(M)\right|=\mid(\zeta-\lambda)$ $g(M)-h(M)|\geqq| \zeta-\lambda|| g(M)|-\|h\|=| \zeta-\lambda|/| g^{-1}(M) \mid-\|h\|$ since $1=\left|g^{-1} g(M)\right|=\left|g^{-1}(M)\right| \cdot|g(M)|$. Since $-\|h\|=\left(-\mu_{\lambda}+\epsilon\right) /\left\|g_{-1}\right\|$, 


$$
\begin{aligned}
\mid(x-z)(M) & \geqq\left[\left(|\zeta-\lambda|\left\|g^{-1}\right\| /\left|g^{-1}(M)\right|\right)-\mu_{\lambda}+\epsilon\right] /\left\|g^{-1}\right\| \\
& \geqq\left[|\zeta-\lambda|-\mu_{\lambda}+\epsilon\right] /\left\|g^{-1}\right\|>0 .
\end{aligned}
$$

Hence, $(x-z)^{-1} f(x)$ is defined and analytic for all $x$ in $F$ and

$$
\begin{aligned}
\frac{1}{2 \pi i} \int_{K \lambda}(x-z)^{-1} f(x) d x= & \frac{1}{2 \pi i} \int_{K_{2}(z)}(x-z)^{-1} f(x) d x \\
& -\frac{1}{2 \pi i} \int_{K_{1}(z)}(x-z)^{-1} f(x) d x
\end{aligned}
$$

by the Cauchy theorem. The integral on the left is equal to $f(z)$ by the Cauchy formula and the right side equals $\phi(z)$. This proves (3), that is, $\phi(z)$ is an analytic continuation of $f(z)$ from the set $D$ to the set $U$. We shall show that it is possible to continue beyond $U$.

If $M$ is in $\mathfrak{M}$, let $E(M)$ be the open annular ring in the complex plane bounded by the circles $K_{1}(M):\left\{z_{0}(M)+r_{1} \exp i t, 0 \leqq t \leqq 2 \pi\right\}$ and $K_{2}(M)$ : $\left\{z_{0}(M)+r_{2}\right.$ exp $\left.i t, \quad 0 \leqq t \leqq 2 \pi\right\}$. Let $Q_{M}=\{z \mid z(M) \in E(M)\}$ and let $Q=\cap_{M \in \mathfrak{M}} Q_{M}$. Then we prove

Lemma 6.1. If $z$ is in $Q$, there is a $\delta>0$ such that for all $x$ on the curves $K_{1}:\left\{z_{0}+\left(r_{1} \exp i t\right) e\right\}$ and $K_{2}:\left\{z_{0}+\left(r_{2} \exp i t\right) e\right\}$ and for all $M$ in $\mathfrak{M}$,

$$
|(x-z)(M)|>\delta \text {. }
$$

Proof. First we prove that the function $|x(M)|$ is continuous in the pair $(x, M)$ for all $x$ in $B$ and all $M$ in $\mathfrak{M}$. Let $x_{0}$ be in $B, M_{0}$ in $\mathfrak{M}, \epsilon>0$. Let $\mathfrak{M}_{0}=\left\{M|| x_{0}(M)-x_{0}\left(M_{0}\right) \mid<\epsilon / 2\right\}$ and $\mathfrak{N}_{0}=\left\{x \mid\left\|x-x_{0}\right\|<\epsilon / 2\right\}$. The product $\mathfrak{N}_{0} \times \mathfrak{M}_{0}$ is a neighborhood of $\left(x_{0}, M_{0}\right)$ in $B \times \mathfrak{M}$. If $(x, M)$ is in $\mathfrak{R}_{0} \times \mathfrak{M}_{0}$, then $|x(M)|-\left|x_{0}\left(M_{0}\right)\right| \leqq\left|x(M)-x_{0}\left(M_{0}\right)\right| \leqq\left|x(M)-x_{0}(M)\right|+\left|x_{0}(M)-x_{0}\left(M_{0}\right)\right|$ $\leqq\left\|x-x_{0}\right\|+\epsilon / 2<\epsilon / 2+\epsilon / 2=\epsilon$. Similarly, $\left|x_{0}\left(M_{0}\right)\right|-|x(M)|<\epsilon$, which establishes the continuity at $\left(x_{0}, M_{0}\right)$.

Now, since $K_{1}$ and $K_{2}$ are compact sets, their union $K_{1} \cup K_{2}$ is also compact. $\mathfrak{M}$ is a compact space $((3))$. Therefore, the product $\left(K_{1} \cup K_{2}\right) \times \mathfrak{M}$ is compact. Since the function $|(x-z)(M)|$ is continuous in the pair $(x, M)$, it assumes a minimum value $\delta \geqq 0$ on $\left(K_{1} \cup K_{2}\right) \times \mathfrak{M}$. If $\delta=0$, there is a pair $\left(x^{\prime}, M^{\prime}\right)$ such that $\left|\left(x^{\prime}-z\right)\left(M^{\prime}\right)\right|=0$. Thus $\left|z\left(M^{\prime}\right)\right|=\left|x^{\prime}\left(M^{\prime}\right)\right|$, contradicting the assumption that $z$ is in $Q$.

The above lemma shows that $Q$ is an open set, for if $z$ is in $Q$, consider the sphere $\left\|z^{\prime}-z\right\|<\delta / 2$. Then $\left|z^{\prime}(M)-z(M)\right| \leqq\left\|z^{\prime}-z\right\|<\delta / 2$ so that $z^{\prime}(M)$ is in $E(M)$ for all $M$; i.e. $z^{\prime}$ is in $Q$.

Obviously, $U \subset Q$ since $z$ in $U$ implies that $r_{1}<\left|z(M)-z_{0}(M)\right|<r_{2}$ for all $M$. To give some idea of the structure of $Q$, we point out that the sphere 
$\left\|z-z_{0}\right\| \leqq r_{1}$ does not intersect $Q$ since $\left|z(M)-z_{0}(M)\right| \leqq r_{1}$ for all such $z$. On the other hand, the set of $z$ such that $\left\|z-z_{0}\right\| \geqq r_{2}$ may intersect $Q$, although $Q$ contains no element of the form $z_{0}+\lambda e$, where $|\lambda|>r_{2}$ since $\mid z_{0}(M)+\lambda$ $-z_{0}(M) \mid>r_{2}$. In fact, if $z=z_{0}+\lambda e+h$ where $|\lambda|>r_{2}$ and $\|h\|<|\lambda|-r_{2}$, then $z$ is not in $U$, for $\left|\left(z-z_{0}\right)(M)\right|=|\lambda+h(M)| \geqq|\lambda|-\|h\|>r_{2}$. Examples may be adduced to show that $Q$ may contain $U$ properly.

Consider the Banach space $(m)$ of bounded sequences $z=\left\{\zeta_{n}\right\}, \zeta_{n}$ a complex number, $\|z\|=\sup _{n}\left\{\left|\zeta_{n}\right|\right\}$. If multiplication of sequences is defined by $\left\{\xi_{n}\right\} \cdot\left\{\zeta_{n}\right\}=\left\{\xi_{n} \zeta_{n}\right\}$ it is easy to see that $(m)$ becomes a $B$-algebra. Let $z_{0}=\{0,0, \cdots\}$. In this case, the set $E$ is a subset of the cartesian product of $\boldsymbol{\aleph}_{0}$ annular rings of the complex plane, each of inner radius $r_{1}$, outer radius $r_{2}$, and center at the origin. Let $b$ be the element of $(m)$ defined by the sequence $\left\{(-1)^{n+1}\left(r_{1}+r_{2}\right) / 2\right\}$. We shall prove that $b$ is in $Q$ but not in $U$.

Note first that if $z=\left\{\zeta_{n}\right\}$ and $z(M)=\lambda$ for some maximal ideal, then there is a sub-sequence of $\left\{\zeta_{n}\right\}$ which converges to $\lambda$. (The sub-sequence may of course consist of only one distinct number $\lambda$.) Otherwise $(z-\lambda e)^{-1}$ $=\left\{1 /\left(\zeta_{n}-\lambda\right)\right\}$ and $\lambda$ is not in the spectrum of $z$. This sub-sequence determines a sequence of maximal ideals $\left\{M_{k}\right\}$ in $(m)$ such that $\lim _{k} z\left(M_{k}\right)=\lambda$. Note also that the set $M_{p}$ of all elements $z=\left\{\zeta_{n}\right\}$ such that $\zeta_{p}=0$ is a maximal ideal. If $a=\left\{\alpha_{n}\right\}$ and $c=\left\{\gamma_{n}\right\}$ are in $M_{p}$, and if $x=\left\{\xi_{n}\right\}, y=\left\{\eta_{n}\right\}$ are arbitrary elements of $(m)$, then $x a+y c=\left\{\xi_{n} \alpha_{n}+\eta_{n} \gamma_{n}\right\}$ is in $M_{p}$ since $\alpha_{p}=\gamma_{p}=0$. This makes $M_{p}$ an ideal. Clearly, $M_{p} \neq(m)$. Suppose $M_{p} \subset I$ where $I$ is a proper ideal of $(m)$, and let $x$ be in $I$ but not in $M_{p}$. Then $x=\left\{\xi_{n}\right\}$ where $\xi_{p} \neq 0$ and $\left|\xi_{n}\right| \leqq\|x\|$ for all $n$. Consider the element $y=\left\{\eta_{n}\right\}$ where $\eta_{p}=0$ and $\eta_{n}=\|x\|+1$ for $n \neq p$. Since $y$ is in $M_{p}, x+y=\left\{\xi_{n}+\eta_{n}\right\}$ is in $I$. But $\left|\xi_{n}+\eta_{n}\right|$ $\geqq\left|\eta_{n}\right|-\left|\xi_{n}\right| \geqq\|x\|+1-\|x\|=1$ for $n \neq p$ and $\left|\xi_{p}+\eta_{p}\right|=\left|\xi_{p}\right| \neq 0$. Thus $(x+y)^{-1}$ exists and $I=(m)$. This contradiction proves $M_{p}$ is a maximal ideal. For any element $x=\left\{\xi_{n}\right\}, x\left(M_{p}\right)=\xi_{p}$ since $x-\xi_{p} e=\left\{\xi_{n}-\xi_{p}\right\}$ is in $M_{p}$.

These remarks show that $b(M)= \pm\left(r_{1}+r_{2}\right) / 2$ for any maximal ideal $M$ in $(m)$. Therefore, $b$ is in $Q$. Now suppose $b$ is in $U$. Since $z_{0}=\{0,0, \cdots\}$, $b=\lambda e+h$ where $r_{1}<|\lambda|<r_{2}$ and $\|h\|<\mu_{\lambda}$. This implies that $|(b-\lambda e)(M)|$ $\leqq\|h\|<\mu_{\lambda}$ for all $M$, or $\max _{n}\left\{\left|\left[(-1)^{n+1}\left(r_{1}+r_{2}\right) / 2\right]-\lambda\right|\right\}<\mu_{\lambda}$. For odd $n$ this means that $\lambda$ lies within the circle of radius $\left(r_{2}-r_{1}\right) / 2$ and center at $\left(r_{1}+r_{2}\right) / 2$, whereas for even $n, \lambda$ must lie within the circle of radius $\left(r_{2}-r_{1}\right) / 2$ and center at $-\left(r_{1}+r_{2}\right) / 2$. This is manifestly impossible so that $b$ is not in $U$.

Returning to the abstract algebra $B$, we observe that $Q$, as an open set, is the union of maximal open connected sets, its components. Let $Q_{1}$ be the component containing $U$. We shall prove that it is possible to continue analytically from $U$ to $Q_{1}$.

THEOREM 6.2. Let $f(z)$ be the function of Theorem 6.1. If $z$ is in $Q_{1}$, then there are curves $K_{1}(z):\left\{z_{0}+\left(\rho_{1} \exp i t\right) e\right\}$ and $\left.K_{2}(z):\left\{z_{0}+\rho_{2} \exp i t\right) e\right\}$ such that 
(1) $\quad \psi(z)=(1 / 2 \pi i) \int_{R_{2}(z)}(x-z)^{-1} f(x) d x-(1 / 2 \pi i) \int_{R_{1}(z)}(x-z)^{-1} f(x) d x$

is a well-defined single-valued analytic function in $Q_{1}$,

$$
\psi(z) \text { is an analytic continuation of } \phi(z) \text { from } U \text { to } Q_{1} \text {. }
$$

Proof. Lemma 6.1 shows that $z(M)$ is contained in the open annulus bounded by the curves $\left\{z_{0}(M)+\left(r_{2}-\delta\right) \exp i t\right\}$ and $\left\{z_{0}(M)+\left(r_{1}+\delta\right) \exp i t\right\}$ for all $M$. Thus if $\rho_{1}$ and $\rho_{2}$ are chosen so that $r_{1}<\rho_{1}<r_{1}+\delta$ and $r_{2}-\delta<\rho_{2}<r_{2}$, then the curves $K_{1}(z)$ and $K_{2}(z)$ are such that $(x-z)(M) \neq 0$ for all $x$ in $K_{1}(z) \cup K_{2}(z)$ and all $M$; i.e. $(x-z)^{-1}$ exists and the integrands in (1) are defined. For any other curves chosen in the prescribed way, the integrals have the same value by the Cauchy theorem. Thus $\psi(z)$ is uniquely determined by $z$.

$\psi(z)$ obviously coincides with $\phi(z)$ for $z$ in $U$. To show that it is analytic in $Q$, we let $\left\|z^{\prime}-z\right\|<\delta / 2$. Then $\left|\left(z^{\prime}-x\right)(M)\right|=\left|\left(z^{\prime}-z\right)(M)+(z-x)(M)\right|$ $\geqq|(z-x)(M)|-\left|\left(z^{\prime}-z\right)(M)\right|>\delta-\delta / 2>0$ for all $x$ in $K_{1}(z) \cup K_{2}(z)$ and all $M$ in $\mathfrak{M}$. If we choose $\rho_{1}^{\prime}$ and $\rho_{2}^{\prime}$ such that $r_{1}<\rho_{1}^{\prime}<r_{1}+\delta / 2, r_{2}-\delta / 2<\rho_{2}^{\prime}<r_{2}$, then the curves $K_{j}\left(z^{\prime}\right):\left\{z_{0}+\left(\rho_{j}^{\prime} \exp i t\right) e\right\}, j=1,2$, will not only serve to define $\psi\left(z^{\prime}\right)$ but $\psi(z)$ as well. Thus we may write

$$
\begin{aligned}
\psi\left(z^{\prime}\right)-\psi(z)= & \frac{1}{2 \pi i} \int_{K_{2}(z)}\left[\left(x-z^{\prime}\right)^{-1} f(x)-(x-z)^{-1} f(x)\right] d x \\
& -\frac{1}{2 \pi i} \int_{K_{1}\left(z^{\prime}\right)} f(x)\left[\left(x-z^{\prime}\right)^{-1}-(x-z)^{-1}\right] d x \\
= & \frac{1}{2 \pi i} \int_{K_{2}\left(z^{\prime}\right)} f(x)\left[\left(z^{\prime}-z\right)\left(x-z^{\prime}\right)^{-1}(x-z)^{-1}\right] d x \\
& -\frac{1}{2 \pi i} \int_{K_{1}\left(z^{\prime}\right)} f(x)\left[\left(z^{\prime}-z\right)\left(x-z^{\prime}\right)^{-1}(x-z)^{-1}\right] d x .
\end{aligned}
$$

A classical argument then shows that $\psi^{\prime}(z)=(1 / 2 \pi i) \int_{K_{2}\left(z^{\prime}\right)}(x-z)^{-2} f(x) d x$ $-(1 / 2 \pi i) \int_{K_{1}\left(z^{\prime}\right)}(x-z)^{-2} f(x) d x$. Thus $\psi(z)$ is an analytic continuation of $\phi(z)$ to $Q_{1}$. The question of whether $Q_{1}$ is the maximal region to which $\phi(z)$ may be continued analytically will be answered negatively by means of an example to be cited in $\$ 7$.

7. Singularities of analytic functions. Let $f(z)$ be a complete analytic function and let $P_{1}\left(z \mid z_{1}\right)$ be a power series functional element of $f(z)$ at the point $z_{1}=z_{0}+\lambda g$, where $g$ is in $G$. Let $K$ be a path in the $\left(z_{0}+\lambda g\right)$-plane joining $z_{1}$ to $z_{0}$ and such that $P_{1}\left(z \mid z_{1}\right)$ may be continued analytically along $K$ up to but not including $z_{0}$. Thus, $z_{0}$ is a $K$-singularity of $f(z)$.

Definition 7.1. Let $z_{0}$ be as above. $z_{0}$ is a " $g$-plane singularity" of $f(z)$ if 
there is an $r_{2}>0$ and a connected arc $K^{\prime}$ of $K$ beginning at $z_{0}$ and contained in the set $E=\left\{z_{0}+\lambda g, 0<|\lambda|<r_{2}\right\}$ with the following property: If $x$ is in $K^{\prime}$ and $P(z \mid x)$ is the power series functional element at $x$, then $P(z \mid x)$ may be continued along every path in $E$ which does not contain $z_{0}$. If $g=e$, we say that $z_{0}$ is a "plane singularity" of $f(z)$. In the continuation process within $E$, it may occur that for each point, $z_{0}+\lambda g$, of $E$ all functional elements whose regions of analyticity contain $z_{0}+\lambda g$ assume the same value at this point. In this case, we say that $z_{0}$ is of "locally single-valued type."

If $z_{0}$ is of locally single-valued type, then $f(z)$ has a functional element whose region of analyticity contains $E$. By Theorem 6.1 this functional element has a Laurent expansion at $z_{0}$ which represents the functional element at least in $E$.

Definition 7.2. The series of negative powers of the Laurent expansion is its "principal part." If the Laurent expansion at a $g$-plane singularity $z_{0}$ has only a finite number of negative powers, then $z_{0}$ is called a "pole." If there are an infinite number of negative powers, $z_{0}$ is an "essential singularity."

For the remainder of this paper, we assume that all complete analytic functions are single-valued unless the contrary is specifically stated. Thus all $g$-plane and plane singularities are of single-valued type and there are Laurent expansions at all such points.

If $z_{0}$ is a plane singularity of $f(z)$, then it is also a $g$-plane singularity for all $g$ in $G_{1}$ and conversely. In proof, the series of negative powers of the Laurent expansion, $f(z)=\sum_{-\infty}^{\infty} b_{n}\left(z-z_{0}\right)^{n}$, converges for $\left\|\left(z-z_{0}\right)^{-1}\right\|<\infty$ (see Theorem 6.1), and the series of positive powers for $\left\|z-z_{0}\right\|<r_{2}$ if $z_{0}$ is a plane singularity. Hence the series converges for all $z=z_{0}+\lambda g$ where $0<|\lambda|<r_{2} /\|g\|$ and $g$ is in $G_{1}$. Thus $z_{0}$ is also a $g$-plane singularity. The converse is proven similarly. (If $g$ is in $G$ but not in $G_{1}$, the Laurent expansion is defined at $z_{0}+\lambda g, 0<|\lambda|<r_{2} /\|g\|$ but may represent a different complete analytic function if the region of definition of the Laurent series consists of disjoint components.)

A plane singularity need not be isolated.

THEOREM 7.1. Let $B$ be a Banach algebra such that (i) the set of regular elements $G$ is dense in $B$ and (ii) the radical of $B$ is $\{0\}$. If $z_{0}$ is a plane singularity of the single-valued complete analytic function $f(z)$, then every sphere $\left\|z-z_{0}\right\|<\rho$ contains a point $z^{\prime}$ which is not contained in the domain of $f(z)$.

Proof. Let $\sum_{-\infty}^{\infty} b_{n}\left(z-z_{0}\right)^{n}$ be the Laurent expansion of $f(z)$ at $z_{0}$. By Theorem 6.1, the Laurent expansion is defined for all $z$ such that $\left(z-z_{0}\right)^{-1}$ exists and $\left\|z-z_{0}\right\|<r_{2}$. Since $f(z)$ is single-valued, $f(z)=\sum_{-\infty}^{\infty} b_{n}\left(z-z_{0}\right)^{n}$ for all such $z$. Since $z_{0}$ is a plane singularity, for some negative integer $-m$, $b_{-m} \neq 0$. Since the radical of $B$ is $\{0\}$, there is an $M^{*}$ in $\mathfrak{M}$ such that $b_{-m}\left(M^{*}\right)$ $\neq 0$.

Let $z_{1}$ be an element of $M^{*}$ such that $\left\|z_{1}\right\|<\rho$. There are such elements 
since $M^{*}$ contains $\lambda z$ whenever it contains $z$. Letting $z^{\prime}=z_{0}+z_{1}$, we have $\boldsymbol{z}^{\prime}\left(M^{*}\right)=z_{0}\left(M^{*}\right)$ and $\left\|z^{\prime}-z_{0}\right\|<\rho$. Suppose $z^{\prime}$ is in the domain of $f(z)$. Then there is a Taylor expansion $\sum_{0}^{\infty} a_{n}\left(z-z^{\prime}\right)^{n}$ which converges in a sphere $\left\|z-z^{\prime}\right\|<\delta$, and equals $f(z)$ therein.

Since $G$ is dense in $B$, there is an element $g$ in $G$ such that $\left\|g-z_{1}\right\|<\epsilon$, where $0<\epsilon<\delta$, but $\epsilon$ is otherwise arbitrary. Let $h=z_{0}+g$. Since $\left\|h-z^{\prime}\right\|$ $=\left\|g-z_{1}\right\|<\epsilon<\delta$, we have $f(h)=\sum_{0}^{\infty} a_{n}\left(h-z^{\prime}\right)^{n}$. Thus $[f(h)]\left(M^{*}\right)=\sum_{0}^{\infty} a_{n}\left(M^{*}\right)$ $\left[h\left(M^{*}\right)-z_{0}\left(M^{*}\right)\right]^{n}$. Now, $\left(h-z_{0}\right)^{-1}$ exists. Furthermore, $\left\|h-z_{0}\right\| \leqq\left\|h-z^{\prime}\right\|$ $+\left\|z^{\prime}-z_{0}\right\|<\epsilon+\rho$. Since $\rho$ and $\epsilon$ may be chosen arbitrarily small, we may write $\left\|h-z_{0}\right\|<r_{2}$. Therefore, the Laurent expansion is defined for $h$ and $f(h)=\sum_{-\infty}^{\infty} b_{n}\left(h-z_{0}\right)^{n}$. It follows that $[f(h)]\left(M^{*}\right)=\sum_{-\infty}^{\infty} b_{n}\left(M^{*}\right)\left[h\left(M^{*}\right)\right.$ $\left.-z_{0}\left(M^{*}\right)\right]^{n}=\sum_{0}^{\infty} a_{n}\left(M^{*}\right)\left[h\left(M^{*}\right)-z_{0}\left(M^{*}\right)\right]^{n}$ where $b_{-m}\left(M^{*}\right) \neq 0$. The equality of the two series holds for a set of complex numbers approaching $h\left(M^{*}\right)$ as a limit. To show this, we set $h_{t}=z_{0}+t g, t$ real. Then $\left\|h_{t}-z^{\prime}\right\|=\left\|t g-\left(z^{\prime}-z_{0}\right)\right\|$ $\leqq\|t g-g\|+\left\|g-\left(z^{\prime}-z_{0}\right)\right\|<|t-1| \cdot\|g\|+\epsilon<\delta$ for $t$ sufficiently close to 1 . It follows that $f\left(h_{t}\right)=\sum_{0}^{\infty} a_{n}\left(h_{t}-z^{\prime}\right)^{n}$. Further, $h_{t}(M)-z_{0}(M)=\operatorname{tg}(M) \neq 0$ for all $M$ and $\left\|h_{t}-z_{0}\right\| \leqq\left\|h_{t}-z^{\prime}\right\|+\left\|z^{\prime}-z_{0}\right\|<|t-1|\|g\|+\epsilon+\rho<r_{2}$ for $t$ close to 1 . Thus, $f\left(h_{t}\right)=\sum_{-\infty}^{\infty} b_{n}\left(h_{t}-z_{0}\right)^{n}$. Finally, $\left[f\left(h_{t}\right)\right]\left(M^{*}\right)=\sum_{0}^{\infty} a_{n}\left(M^{*}\right)\left[h_{t}\left(M^{*}\right)\right.$ $\left.-z_{0}\left(M^{*}\right)\right]^{n}=\sum_{-\infty}^{\infty} b_{n}\left(M^{*}\right)\left[h_{t}\left(M^{*}\right)-z_{0}\left(M^{*}\right)\right]^{n}$ for $|t-1|<\epsilon_{1}$, say. As $t$ approaches $1, h_{t}\left(M^{*}\right)$ approaches $h\left(M^{*}\right)$. Applying the classical identity theorem for complex analytic functions, we conclude that $a_{n}\left(M^{*}\right)=b_{n}\left(M^{*}\right)$ for all $n,-\infty<n<\infty$ (uniqueness of Laurent expansion). This implies that $b_{-m}\left(M^{*}\right)=0$ which is a contradiction. Therefore, $z^{\prime}$ is not in the domain of $f(z)$.

The method of proof of the preceding theorem shows that a weaker hypothesis than $G$ being dense in $B$ would suffice. It is sufficient that $B$ be such that every maximal ideal $M$ contains an element $b$ on the boundary of $G$. Then we may set $z_{1}=\lambda b$ if $\lambda$ is chosen in the right way, and the existence of a regular element in any neighborhood of $z_{1}$ is assured.

We shall give examples of algebras in which $G$ is a dense set and examples of algebras in which $G$ is nondense. In fact, there is an algebra having a maximal ideal consisting entirely of elements which are in the interior of the set of singular elements.

The algebra of bounded sequences $(m)$ is a $B$-algebra in which $G$ is a dense set. The set of singular elements consists of all sequences $\left\{\sigma_{n}\right\}$ such that $\inf _{n}\left\{\left|\sigma_{n}\right|\right\}=0$. If a ${ }^{*}$-operation is introduced into $(m)$ by defining $\left\{\sigma_{n}\right\}^{*}$ $=\left\{\bar{\sigma}_{n}\right\}$, where $\bar{\sigma}_{n}$ is the complex conjugate of $\sigma_{n}$, then $(m)$ becomes a $B^{*}$ algebra with real bounded *-operation [10] since $\left\{\sigma_{n}\right\}^{*}=\left\{\sigma_{n}\right\}$ if and only if $\sigma_{n}$ is real for all $n$, i.e. the spectrum of $\left\{\sigma_{n}\right\}$ is real, and $\sup _{x \in(m)}\left\{\left\|x^{*}\right\| /\|x\|\right\}$ $=1$ where $\left\|x^{*}\right\|=\sup _{n}\left\{\left|\bar{\sigma}_{n}\right|\right\}$. It follows from a theorem of Rickart [11] that $(m)$ is a proper algebra; i.e. every singular element is a generalized zerodivisor. ( $z$ is a generalized zero-divisor if there is a sequence of elements $z_{n}$ with $\left\|z_{n}\right\|=1$ and such that $\lim _{n} z z_{n}=0$.) Furthermore, the set of singular ele- 
ments is contained in the closure of $G$, for if $\left\{\sigma_{n}\right\}$ is singular and $\epsilon>0$, there is a subsequence $\left\{\sigma_{n_{j}}\right\}$ such that $\left|\sigma_{n_{j}}\right|<\epsilon / 2$ and for $n \neq n_{j},\left|\sigma_{n}\right| \geqq \epsilon / 2$. Let $\gamma_{n}=\sigma_{n}$ for $n \neq n_{j}$ and otherwise let $\gamma_{n_{j}}=\epsilon / 4$. The element defined by the sequence $\left\{\gamma_{n}\right\}$ is regular since $\left|\gamma_{n}\right| \geqq \epsilon / 4$ for all $n$. Also $\left\|\left\{\gamma_{n}\right\}-\left\{\sigma_{n}\right\}\right\|$ $=\sup _{n}\left\{\left|\gamma_{n}-\sigma_{n}\right|\right\}<\epsilon$. Thus every neighborhood of $\left\{\sigma_{n}\right\}$ intersects $G$. Hence, every singular element is in the closure of $G$ so that $(m)$ is in the closure of $G$. Thus $G$ is dense in $(m)$.

In the same way it is shown that the sub-algebra of convergent sequences is also such that the regular elements in it form a dense set.

The algebra $C[0,1]$ of continuous functions (complex-valued) on the unit interval $[0,1]$ with the norm defined by $\|f(t)\|=\max _{0 \leqq t \leqq 1}\{|f(t)|\}$ is another example of an algebra in which the regular elements form a dense set. In proof, let $f(t)$ be a singular element. Then $\min _{t}\{|f(t)|\}=0$. Let $T=\{t|| f(t) \mid \leqq \epsilon / 3\}$ where $\epsilon<\max _{t}\{|f(t)|\}$. We define the function $g(t)$ as follows: for $t$ not in $T, g(t)=f(t)$. If $t \in T$ and $|f(t)|=\epsilon / 3$ then $g(t)=f(t)$. If $t$ is in $T$ and $|f(t)|<\epsilon / 3$, then there is an open interval containing $t$ and such that $|f(t)|<\epsilon / 3$ for all points in this interval. Let $\left(t_{1}, t_{2}\right)$ be the largest such interval. By continuity, $\left|f\left(t_{1}\right)\right|=\left|f\left(t_{2}\right)\right|=\epsilon / 3$. Let $f\left(t_{1}\right)=(\epsilon / 3)$ exp $i \theta_{1}$ and $f\left(t_{2}\right)=(\epsilon / 3)$ exp $i \theta_{2}$. We define $g(t)=(\epsilon / 3)$ exp $\left[i u \theta_{2}+i(1-u) \theta_{1}\right]$ where $u=\left(t-t_{1}\right) /\left(t_{2}-t_{1}\right)$ for all $t$ in $\left(t_{1}, t_{2}\right)$. This describes $g(t)$ completely. Clearly, it is a continuous function. It is a regular element of $C[0,1]$ since $\inf _{t}\{|g(t)|\}$ $=\epsilon / 3>0$. Since $\max _{t}\{|g(t)-f(t)|\}<\epsilon, g(t)$ is in an $\epsilon$-neighborhood of $f(t)$. Thus every singular element is in the closure of the set of regular elements which therefore form a dense set.

We now consider another example. Let $E$ denote the sub-algebra of $C[0,1]$ generated by the element $b=\exp 2 \pi i t$ and the complex numbers. $E$ consists of all polynomials in $b$ (with complex coefficients) and their limits (uniform convergence). The spectrum of $b$ consists of all the complex numbers $\lambda$ with $|\lambda| \leqq 1$. In proof, we note that for $\lambda<1$, we have

$$
\frac{1}{\lambda+\exp (2 \pi i t)}=\left(\frac{1}{\exp (2 \pi i t)}\right)\left[1-\lambda / \exp (2 \pi i t)+\lambda^{2} /(\exp 2 \pi i t)^{2}-\cdots\right]
$$

and this element is not in $E$ for if we consider the function $g(t)=[1 /(\lambda+\exp 2 \pi i t)$ $\left.-\sum_{0}^{{ }_{0}} \lambda_{n} \exp 2 \pi i n t\right]$ we find that $\|g(t)\| \geqq 1$. We have $1=\left|\int_{0}^{1}(\exp 2 \pi i t) g(t) d t\right|$ $\leqq \max _{t}\{|g(t)|\}=\|g(t)\|$. Finally, for $|\lambda|=1$, there exists $t_{0}$ such that

$$
\exp 2 \pi i t_{0}=\lambda
$$

so that $(\exp 2 \pi i t-\lambda)^{-1}$ does not exist even in $C[0,1]$. Since 0 is in the spectrum of $b, b$ is a singular element.

Now we show that the principal ideals of the form $(b-\lambda)$, where $|\lambda|<1$, are maximal. If $|\lambda|<1$, the element $b-\lambda$ is singular and the principal ideal $(b-\lambda)$ is contained in a maximal ideal, $M$. Then $b(M)=\lambda$. Now suppose $z$ is in $M$ and let $P_{n}(b)=\sum_{k=0}^{\gamma_{n}} \alpha_{k n} b^{k}, n=1,2, \cdots$, be a sequence of polynomials 
such that $\lim _{n} P_{n}(b)=z$. Let $q_{m}$ be an integer such that $\left\|P_{q_{m}}(b)-z\right\|<1 / m$.

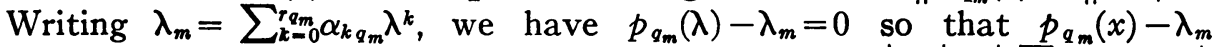
$=(x-\lambda) g_{q_{m}}(x)$, where $g_{q_{m}}(x)$ is a polynomial in $x .\left|\lambda_{m}\right|=\left|\sum_{k=0}^{r q_{m}} \alpha_{k q_{m}} \lambda^{k}\right|$ $=\left|P_{q_{m}}(b)(M)-z(M)\right| \leqq \| P_{q_{m}}(b)-z||<1 / m$. Thus $\lim _{m \rightarrow \infty}\left[P_{q_{m}}(b)-\lambda_{m}\right]$ $=\lim _{m \rightarrow \infty} P_{q_{m}}(b)-\lim _{m} \lambda_{m}=\lim _{m} P_{q_{m}}(b)=z . \operatorname{But}_{\lim _{m}}\left[P_{q_{m}}(b)-\lambda_{m}\right]=\lim _{m}(b-\lambda)$ $\cdot g_{q_{m}}(b)=(b-\lambda) \lim _{m} g_{q_{m}}(b)$ and we have $z=(b-\lambda) c$, where $c=\lim _{m} g_{q_{m}}(b)$ is in $E$. Thus, $M$ is contained in the principal ideal $(b-\lambda)$, which means that $M=(b-\lambda)$.

Now let us consider the maximal ideal $(b)=(\exp 2 \pi i t)$ in $E$. If $z_{0}$ is in $(b)$, then $z_{0}=b \lim _{n} p_{n}(b)$ where $p_{n}(b)$ is a polynomial in $b$ for $n=1,2, \cdots$. The element $z_{0}$ is obviously singular in $E$. If $z_{0} \neq 0$, we shall show that there is a spherical neighborhood of $z_{0}$ contained in the set of singular elements.

Since $z_{0}$ is singular and not zero, there is an $\epsilon, 0<\epsilon<1$, such that the sphere $\left\|z-z_{0}\right\|<\epsilon$ contains no scalars. We shall show that all $z$ in this sphere are singular, i.e. all the nonzero elements in $(b)$ are interior points of the set of singular elements. Thus, the regular elements are certainly not dense in this algebra.

Choose $\delta$ such that $0<\epsilon+\delta<1$. Since $z$ is not a scalar, there is a polynomial $\sum_{0}^{r} \lambda_{k} b^{k}$ with $\lambda_{r} \neq 0, r \geqq 1$, such that $\left\|z-\sum_{0}^{r} \lambda_{k} b^{k}\right\|<\delta$. Further, since $z_{0}=b \lim _{n} p_{n}(b)$, given $\gamma, 0<\epsilon+\delta+\gamma<1$, we can find $n$ such that $\left\|z_{0}-b p_{n}(b)\right\|$ $<\gamma$. Writing $p_{n}(b)=\sum_{0}^{s} \alpha_{k} b^{k}$, we have

$$
\left\|\sum_{0}^{\infty} \lambda_{k} b^{k}-\sum_{0}^{r} \alpha_{k} b^{k+1}\right\|<\epsilon+\delta+\gamma<\mu<1 .
$$

Hence, for any $M$,

$$
\left|\lambda_{0}+\left(\lambda_{1}-\alpha_{0}\right) b(M)+\left(\lambda_{2}-\alpha_{1}\right) b^{2}(M)+\cdots\right|<\mu .
$$

Since 0 is in the spectrum of $b$, there is a maximal ideal such that $b(M)=0$ (i.e. $M=(b))$. Hence, $\left|\lambda_{0}\right|<\mu$. This implies that $\sum_{0}^{r} \lambda_{k} b^{k}$ is singular, for the complex polynomial $\sum_{0}^{r} \lambda_{k} x^{k}$ has a complex root $\left|\zeta_{1}\right|<1$. But $\zeta_{1}$ is in the spectrum of $b$ and there is a maximal ideal $M_{1}$ such that $b\left(M_{1}\right)=\zeta_{1}$ and we have $\left(\sum_{0}^{r} \lambda_{k} b^{k}\right)\left(M_{1}\right)=\sum_{0}^{r} \lambda_{k} \xi_{1}^{k}=0$. Since $\delta$ may be chosen arbitrarily small, it is seen that every neighborhood of $z$ contains singular elements. Hence $z$ cannot be regular and the sphere $\left\|z-z_{0}\right\|<\epsilon<1$ is contained in the set of singular elements.

Incidentally, this also affords an example of a commutative $B$-algebra in which the set of generalized zero-divisors is not contained in the intersection of the closure of $G$ with the set of singular elements [11]. The element $b-e=(\exp 2 \pi i t-1)$ is in this intersection since all elements of the form $(\exp 2 \pi i t-1-\epsilon), \epsilon>0$, in a neighborhood of $(b-e)$ are regular. Rickart [11] shows that every singular element on the boundary of $G$ is a generalized zerodivisor. Hence, the element $(\exp 2 \pi i t-1)$ is a generalized zero-divisor. There- 
fore the element $(\exp 2 \pi i t)(\exp 2 \pi i t-1)$ is also a generalized zero-divisor.

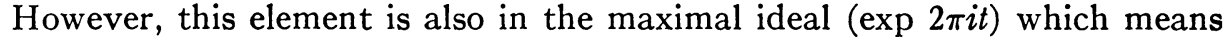
that it is an interior point of the set of singular elements. Hence it is not in the closure of $G$.

In connection with Theorem 7.1 , we remark that the point $z^{\prime}$ may be a planar singularity or not, that is, both situations do arise. As a simple illustration of the first situation, we consider the cartesian product of the complex plane with itself, i.e. the set of pairs $\left(\lambda_{1}, \lambda_{2}\right)$ with $\lambda_{1}$ and $\lambda_{2}$ complex. With the usual definitions of addition and multiplication and now with norm given by $\max \left\{\left|\lambda_{1}\right|,\left|\lambda_{2}\right|\right\}$, this set becomes a $B$-algebra. If we let $a_{1}=(1,0)$ and $a_{0}=(0,1)$, then the function $f(z)=a_{1} z^{-1}+a_{0}$, where $z=\left(\zeta_{1}, \zeta_{2}\right)$, may be written in the form $f(z)=\left(1 / \zeta_{1}, 0 / \zeta_{2}\right)+(0,1)=\left(1 / \zeta_{1}, 1\right)$. The formula $a_{1} z^{-1}+a_{0}$ defines $f(z)$ for all $z$ in $G$ and only such $z$. Thus the set $Q$ of Lemma 6.1 is equal to $G$, which consists of all pairs $\left(\lambda_{1}, \lambda_{2}\right)$ such that $\lambda_{1} \neq 0, \lambda_{2} \neq 0 . f(z)$ may obviously be continued analytically outside of $Q$ by means of the formula $\left(1 / \zeta_{1}, 1\right)$ which is defined for all $z=\left(\zeta_{1}, \zeta_{2}\right)$ with $\zeta_{1} \neq 0$ and coincides with $f(z)$ in $G$. This also is the maximum analytic extension of $f(z)$ and illustrates the possibility of continuing beyond the set $Q$. The point $(0,0)$ is clearly a pole of $f(z)$. It is not isolated since all points $z^{\prime}=\left(0, \zeta_{2}\right)$ with $\zeta_{2} \neq 0$ in a neighborhood of $(0,0)$ are points which cannot be in the domain of the complete analytic function determined by $f(z)$. In fact, these points are also poles.

An example of the second situation is afforded by the algebra $(m)$. Let $f(z)=z^{-1}$ and $z^{\prime}=\{1 / n\}$. The point 0 is obviously a pole and $z^{\prime}$ is not in the domain of the complete analytic function $z^{-1}$. Further, every annulus of the form $\left\{z^{\prime}+\lambda e, 0<|\lambda|<r\right\}$ must contain elements which are singular and therefore not in the domain of $z^{-1}$. Thus $z^{\prime}$ is not a plane singularity. On the other hand, $z^{\prime \prime}=\{0,1,1, \cdots, 1, \cdots\}$ is a pole, so that both kinds of points exist in a neighborhood of the pole 0 .

The behavior of analytic functions in a neighborhood of a singularity is further illuminated by the following analogue of the classical theorem on poles.

TheOREM 7.2. Let $B$ be a Banach algebra such that (i) the set of regular elements is dense in $B$ and (ii) the radical of $B$ is $\{0\}$. If the point $b$ in $B$ is a pole of the single-valued complete analytic function $f(z)$, then $\lim _{z \rightarrow b}\|f(z)\|=\infty$ regardless of the manner in which $z$ approaches $b$.

Proof. Let $\sum_{n=-m}^{\infty} a_{n}(z-b)^{n}$ be the Laurent expansion of $f(z)$ at $b$, assuming that $b$ is a pole of order $m$. By Theorem 6.1 , the Laurent expansion is defined for all $z$ such that $(z-b)^{-1}$ exists and $\|z-b\|<r_{2}$. Since $f(z)$ is singlevalued, $f(z)=\sum_{-m}^{\infty} a_{n}(z-b)^{n}$ for all such $z$, and $(z-b)^{m} f(z)=\left[a_{-m}+a_{-m+1}(z-b)\right.$ $+\cdots]$. We denote the quantity in brackets by $g(z)$. For a constant $p$ there is a $\delta>0$ such that $\|g(z)\| \geqq p>0$, whenever $\|z-b\|<\delta$. This follows from

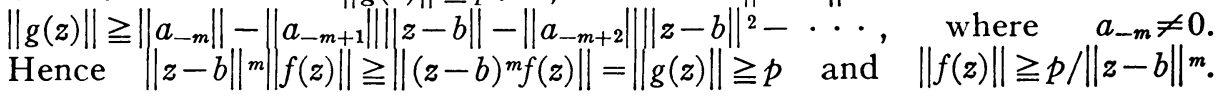


Thus if $z$ approaches $b$ in the region for which the Laurent expansion is valid, then $\|f(z)\| \rightarrow \infty$.

Now suppose $\left\{z_{k}\right\}$ is a sequence of elements in the domain of $f(z)$ for which the Laurent series is not defined and such that $\lim _{k} z_{k}=b$. There is an integer $N$ such that $\left\|z_{k}-b\right\|<r_{2} / 3$ for all $k>N$. For each $k$ there is an element $g_{k}$ in $G$ such that $\left\|g_{k}-\left(z_{k}-b\right)\right\|<\epsilon_{k}$, where the $\left\{\epsilon_{k}\right\}$ is a sequence of decreasing positive numbers approaching 0 . If we let $c_{k}=b+g_{k}$, then $c_{k}-b$ is regular and $f\left(c_{k}\right)=\sum_{n=-m}^{\infty} a_{n}\left(c_{k}-b\right)^{n}$ for $k>N$. Since $\left\|c_{k}-b\right\|=\left\|g_{k}\right\| \leqq\left\|z_{k}-b\right\|+\epsilon_{k}$, we see that $\lim _{k \rightarrow \infty}\left\|g_{k}\right\|=0$. From $\left|g_{k}(M)\right| \leqq\left\|g_{k}\right\|$ it follows that $\lim _{k}\left|\left(c_{k}-b\right)(M)\right|=0$. Since the radical of $B$ is $\{0\}$, there is a maximal ideal $M^{\prime}$ for which $a_{-m}\left(M^{\prime}\right)$ $\neq 0$. Thus $\left[f\left(c_{k}\right)\right]\left(M^{\prime}\right)=\sum_{-m}^{\infty} a_{n}\left(M^{\prime}\right)\left[\left(c_{k}-b\right)\left(M^{\prime}\right)\right]^{n}$ approaches infinity as $k$ approaches infinity. Therefore, $\left\|f\left(c_{k}\right)\right\|$ becomes infinite also. If the $\epsilon_{k}$ are chosen such that $\left\|f\left(c_{k}\right)-f\left(z_{k}\right)\right\|<1$, which is possible by the continuity of $f(z)$ at the points $z_{k}$, it follows that $\left\|f\left(z_{k}\right)\right\|$ approaches infinity also.

The following remark shows that the behavior of functions in a neighborhood of a singularity is not always analogous to the classical behavior. For example, the Weierstrass theorem for essential singularities does not always hold. Again we assume that $G$ is dense in the algebra. If the point 0 is an essential singularity of the single-valued complete analytic function $f(z)$, and $\sum_{-\infty}^{\infty} a_{n} z^{n}$ is its Laurent expansion around 0 , where $a_{n} \neq 0$ for an infinite number of negative $n$, then it may occur that $a_{n}(M)=0$ for all $n<0$ and some maximal ideal $M$. Then $[f(z)](M)=\sum_{0}^{\infty} a_{n}(M)[z(M)]^{n}$ for all $z$ for which the Laurent series is defined. By an argument like the one in the preceding theorem, it can be shown that $[f(z)](M)$ approaches $a_{0}(M)$ as $z$ approaches 0 . Hence, $f(z)$ cannot approach any element of the form $\lambda e$, where $\lambda \neq a_{0}(M)$, in every neighborhood of 0 , as the Weierstrass theorem requires.

To obtain more information on the behavior of functions in a neighborhood of a singularity, we turn to a consideration of rational functions. In preparation, we shall discuss some of the properties of polynomials over a $B$-algebra.

8. Polynomials over a $B$-algebra. Let $p(x)=a_{0}+a_{1} x+\cdots+a_{n} x_{n}$ be a polynomial with coefficients $a_{j}$ in $B$, and leading coefficient $a_{n} \neq 0$. Let $D(p)$ denote the set of elements $z$ in $B$ such that $p(z)$ is in $G$. We note that $D(p)$ may be empty. For example, if there is a maximal ideal $M$ such that $a_{j}(M)=0$ for $j=0,1, \cdots, n$, then $[p(z)](M)=\sum_{j=0}^{n} a_{j}(M)[z(M)]^{j}=0$ for all $z$ in $B$. Hence, $p(z)$ is singular for all $z$ in $B$. Whether this is a necessary condition for $D(p)$ to be empty is not known. In the algebra $(m)$ it is the case, but the proof employed for $(m)$ does not generalize. However, if $D(p)$ is empty, we may state the following.

Lemma 8.1. If $p(x)$ is a polynomial such that $D(p)$ is empty, then the set of complex numbers $\lambda$ such that $\lambda$ is a zero of one of the polynomials $[p(x)](M)$ $=\sum_{j=0}^{n} a_{j}(M) x^{i}$, where $M$ is a maximal ideal, must contain the entire complex plane. 
Proof. Suppose there is a complex number $\lambda$ such that $\lambda$ is not a zero of any polynomial $[p(x)](M)$ for any $M$ in $\mathfrak{M}$. Then $[p(\lambda e)](M)=\sum_{j=0}^{n} a_{j}(M) \lambda^{i}$ $\neq 0$ for all $M$, which means that $\lambda e$ is in $D(p)$, contrary to hypothesis.

The converse of the lemma is not true, as an example shows. Again we consider the algebra $E$ generated by $b=\exp 2 \pi i t$ and the complex numbers. We have shown that the spectrum of $b$ in $E$ consists of all $\lambda$ such that $|\lambda| \leqq 1$. Hence there is a maximal ideal $M_{\lambda}$ such that $b\left(M_{\lambda}\right)=\lambda$ for each $\lambda$ with $|\lambda| \leqq 1$. Let $p(x)$ be the polynomial $b x^{2}+\left(b^{2}-1\right) x-b$. Thus $p(x)=(x+b)$ $\cdot(b x-1)$. If $\zeta$ is any complex number, there is a maximal ideal $M_{\zeta}$ such that $[p(x)]\left(M_{\zeta}\right)$ is a polynomial having $\zeta$ as one of its zeros. In proof, if $|\zeta| \leqq 1$, then there is a maximal ideal $M_{\zeta}$ such that $b\left(M_{\zeta}\right)=-\zeta$ so that $(x+b)\left(M_{\zeta}\right)$ $=(x-\zeta)$ has $\zeta$ as a zero. If $|\zeta|>1$, then $1 /|\zeta|<1$ and there is a maximal ideal $M^{\prime}$ such that $b\left(M^{\prime}\right)=1 / \zeta$ so that $\left[b\left(M^{\prime}\right) x-1\right]$ has $\zeta$ as a zero. Therefore, the set of zeros of all the polynomials $[p(x)](M), M$ in $\mathfrak{M}$, contains every complex number; i.e. $D(p)$ contains no complex number. Yet, $D(p)$ is not empty, for if we let $z=1-b / 2$, then $(b z-1)=(-1 / 2)\left(b^{2}-2 b+2\right)=(-1 / 2)$ $\cdot(b-1+i)(b-1-i)$, so that $(b z-1)(M) \neq 0$ for all $M$. Likewise, $z+b$ $=(1 / 2)(b+2)$ so that $(z+b)(M) \neq 0$ for all $M$. Hence, $(1-b / 2)$ is in $D(p)$. Since $D(p)$ is evidently an open set, it contains infinitely many elements whenever it contains one.

We now state a sufficient condition for $D(p)$ to be nonempty.

LemMA 8.2. If either $a_{n}$ or $a_{0}$ is a regular element, then $D(p)$ is not empty.

Proof. If $a_{0}$ is regular, then since $p(0)=a_{0}$, it follows that $p(z)$ is regular for $z$ near 0 .

If $a_{n}$ is regular, then for any complex $\lambda$ we have $p(\lambda e)=\left[a_{0} a_{n}^{-1} / \lambda^{n}+\cdots\right.$ $\left.+a_{n-1} a_{n}^{-1} / \lambda+1\right] \lambda^{n} a_{n}$. For $|\lambda|$ sufficiently large, $p(\lambda e)$ is close to the regular element $\lambda^{n} a_{n}$ and is therefore regular. Thus $\lambda e$ is in $D(p)$.

The preceding example with $p(x)=b x^{2}+\left(b^{2}-1\right) x-b$ shows that the above sufficient condition for $D(p)$ to be nonempty is not a necessary condition, since $b$ is not a regular element of the algebra $E$.

Let $S(p)$ be the complement of $D(p)$, that is, the set of all $z$ such that $p(z)$ is singular.

LEMMA 8.3. If $a_{0}$ is singular or if one of the $a_{i}$ is not in the radical for $i \neq 0$, then $S(p)$ is not empty.

Proof. There is a maximal ideal $M$ such that $a_{n}(M) \neq 0$. For this $M$, the polynomial $[p(x)](M) \equiv a_{n}(M) x^{n}+\cdots+a_{0}(M)$ has at least one complex zero, $\zeta$. Then $[p(\zeta e)](M)=0$ and $\zeta e$ is in $S(p)$.

Combining the two preceding lemmas, we may state

THEOREM 8.1. If $p(x)$ is a polynomial with leading coefficient $a_{n}$ in $G$, then $p(x)$ separates the algebra $B$ into an open nonempty set $D(p)$ and a nonempty closed set $S(p)$ such that $D(p)$ is the set of all $z$ in $B$ for which $p(z)$ is in $G, S(p)$ 
is its complement, and if at least one $a_{i}$ is not in the radical for $i<n$, both $D(p)$ and $S(p)$ intersect $G$.

Concerning the second lemma, we remark that if $a_{n}$ is in the radical of $B$ and $a_{n} \neq 0$, then $S(p)$ may or may not be empty according as $a_{0}$ is or is not regular. For example, if $p(x)=a_{n} x^{n}+a_{0}$ and $a_{0}$ is in the radical while $a_{0}$ is in $G$, then $[p(z)](M) \neq 0$ for all $z$ in $B$ and all $M$ in $\mathfrak{M}$. Thus $S(p)$ is empty. On the other hand, if $a_{0}$ is not in $G$, then $D(p)$ is empty.

Theorem 8.1 may be regarded as an attempt to obtain something like a fundamental theorem of algebra. However, it affords meager information about the zeros of $p(x)$. Yet, in a sense to be explained, it is a best possible result for abstract algebras.

Let $Z(p)$ denote the set of zeros of $p(x)$. Obviously, $Z(p) \subset S(p)$ and therefore may be empty. However, for $Z(p)$ to be empty it is not necessary that $a_{n}$ be in the radical. In fact, as the following example shows, $a_{n}$ may be regular.

Let $C^{\prime}$ be the sub-algebra of $C[0,1]$ consisting of those functions $f(t)$ for which $f(0)=f(1)$. Let $p(x)$ be the polynomial $a_{0}+a_{2} x^{2}$ where $a_{0}=-e$ and $a_{2}=\exp 2 \pi i t$. Suppose $p(z)=0$. Then $a_{2} z^{2}=-a_{0}$ or $(\exp 2 \pi i t) z^{2}=1$. Thus $z^{2}=\exp (-2 \pi i t)$ and $z= \pm \exp (-\pi i t)$. Since $\exp (-\pi i t)$ is not in $C^{\prime}, p(x)$ has no zeros in $C^{\prime}$. Yet, $a_{2}=\exp 2 \pi i t$ is regular in $C^{\prime}$.

Therefore, for an arbitrary algebra, there can be no fundamental theorem of algebra. At the same time, for some polynomials the set $Z(p)$ may be infinite. In the algebra $(m)$, if $p(x)=a_{n} x^{n}+a_{0}$ where $a_{n}$ is the sequence $\left\{\alpha_{n k}\right\}, k=1,2, \cdots$, and $\inf _{k}\left\{\left|\alpha_{n k}\right|\right\}>0$ and $a_{0}=\left\{\alpha_{0 k}\right\}$ where $\inf _{k}\left\{\left|\alpha_{0 k}\right|\right\}>0$, then $p(x)$ has infinitely many zeros. In $(m)$, if $a_{n}$ is regular, then $p(x)$ has at least one zero.

The foregoing indicates to some extent how the algebraic properties of polynomials over a $B$-algebra differ from those of complex polynomials. We now show that the metric properties differ also.

We recall that for complex polynomials the modulus $|p(x)|>N$ for all $x$ such that $|x|>r, N>0$ being an arbitrary real number. A very simple example shows that this is not so for arbitrary $B$-algebras. In the algebra of pairs $\left(\lambda_{1}, \lambda_{2}\right)$, let $p(x)=a_{1} x+a_{0}$ where $a_{1}=(1,0)$ and $a_{0}=(1,1)$. For every $r>0$ there is a $z$ in $B$ with $\|z\|>r$ and $\|p(z)\|=1$; e.g. $z=(0, r+1)$.

If the polynomial is restricted by the requirement that $a_{n}$ be in $G$, one might expect the classical result to apply. For the algebras $(m)$ and $C[0,1]$, or any algebra in which the norm has the property $\left\|z^{2}\right\|=\|z\|^{2}$, this is true. If $\left\|z^{2}\right\|=\|z\|^{2}$, then $\|z\|=\lim \sup _{n}\left(\left\|z^{n}\right\|\right)^{1 / n}=\max \{|z(M)|, M$ in $\mathfrak{M}\}$. Hence, $\|p(z)\| \geqq|[p(z)](M)| \geqq|z(M)|^{n}\left[\mu-\left\|a_{n-1}\right\| /|z(M)|-\cdots-\left\|a_{0}\right\| /|z(M)|^{n}\right]$ where $\mu=\min \left\{\left|a_{n}(M)\right|, M\right.$ in $\left.\mathfrak{M}\right\}>0$. If $\|z\|>r$, then $\max _{M}\{|z(M)|\}>r$ so that we need only choose $r$ large enough to obtain $\|p(z)\|>N$.

Of course, if $\max _{M}\{|z(M)|\}<\|z\|$, then the proof is invalid, as it is in an algebra with radical, or in the algebra (1) of convergent (absolutely) power 
series with complex coefficients. (1) is an algebra with multiplication and addition defined as ordinary multiplication and addition of series and the norm of a power series defined by the usual formula $\left\|\sum_{0}^{\infty} \alpha_{n} x^{n}\right\|=\sum_{0}^{\infty}\left|\alpha_{n}\right|$. In (1), the element $p(x)=x^{2}-x-2$ has norm $1+1+2=4$ whereas $\max \{|p(x)|,|x| \leqq 1\}=\max \{|[p(x)](M)|, M$ in $\mathfrak{M}\}<4$ as an elementary calculation shows. The behavior of the norm in algebras like (1) is not clear. We point out that for $N>0$ there is an $r$ such that for all $\lambda e$ with $|\lambda|>r$, $\|p(\lambda e)\|>N$ if $a_{n}$ is in $G$. If $F$ is the set of elements $z$ such that $\|p(z)\|>N$, then $F$ contains all $z$ such that $\max _{M}\{|z(M)|\}>r . F$ is a subset of the set of $z$ such that $\|z\|>r$. If the radical of $B$ is not $\{0\}, F$ is a proper subset.

We turn now to a study of rational functions.

9. Rational functions. Let $q(x)=a x+b$, where $a$ is in $G$. If $q(z)=0$, then $a z=-b$, and $z=a^{-1} b$. Thus $q(x)$ has precisely one zero. The rational function $g(x)=[q(x)]^{-1}=a^{-1}\left(x+a^{-1} b\right)^{-1}=a^{-1}(x-c)^{-1}$ is defined for all elements of the form $c+g$ where $-c=a^{-1} b$ and $g$ is in $G$. We denote this set by $D(q)$. It is easily seen that $D(q)$ is homeomorphic to $G$ with components mapping onto components under the translation $g \rightarrow c+g$. Unlike $G$, however, $D(q)$ need not be a group under multiplication.

Let $D(q)^{\prime}$ be the subset of $D(q)$ consisting of elements $c+g$ such that $g$ is in the principal component $G_{1}$. The fundamental group of $D(q)^{\prime}$ is isomorphic to $\pi_{1}\left(G_{1}\right)$, the fundamental group of $G_{1}$. It has been shown [12] that the curves of the form $\{c+\exp t y \mid \exp y=e, 0 \leqq t \leqq 1\}$ form a set of generators with base point $c+e$. Any closed curve in $D(q)^{\prime}$ with initial point $c+e$ is homotopic to one of these generators. Let the curve $K$ in $D(q)^{\prime}$ be parametrized by $z(t)$ where $z(0)=z(1)=c+e$. Let $K(c)$ be the curve in $G$ parametrized by $x(t)$ $=z(t)-c$ so that $x(0)=x(1)=e$. Then $\int_{K}(z-c)^{-1} d z=\int_{K(c)} x^{-1} d x=2 \pi i y$, where $\exp 2 \pi i y=e$. Thus the residues of $(x-c)^{-1}$ are the same as those of $x^{-1}$.

The formula $(x-c)^{-1}$ defines a single-valued complete analytic function in each of the components of $D(q)$. The point $c$ is obviously a pole of order one of each of these functions. However, there may be other simple poles. Suppose $z$ in $B$ is such that the complement of the spectrum of $z-c$ contains the interior of a circle of radius $r$, centered at 0 , with the exception of the point 0 itself. Then $(\lambda e+z-c)^{-1}$ is defined for all $|\lambda|<r$ except $\lambda=0$, so that $z$ is a pole. This is also a necessary condition for an element $z$ to be a pole. There may be an infinitude of poles, possibly nondenumerable. For example, if $B$ is a reducible algebra and $e_{j}$ is an idempotent, then $z_{j}=c+e_{j}$ has the above property since the spectrum of $z_{j}-c$ consists of the points 0 and 1 . There may be a nondenumerable number of idempotents in the algebra. If the radical of $B$ is $\{0\}$, and $B$ is irreducible, then since the spectrum of every element is connected, we may conclude that the only plane singularity of $(x-c)^{-1}$ is the pole $c$.

We observe that all the residues of $x^{-1}$ may be obtained by integrating along special curves which contain the various singularities of $x^{-1}$ as "center" 
in a sense. For example, if $e_{j}$ is an idempotent, then $e_{j}$ is a pole of $x^{-1}$. Let $K\left(e_{j}\right)$ be the curve $\left\{e_{j}+\left(r_{j} \exp 2 \pi i t\right) e, 0<r_{j}<1\right\}$. Then $x^{-1}$ is defined on $K\left(e_{j}\right)$ and for all points of the $e_{j}$-plane interior to $K\left(e_{j}\right)$, and we have

$$
\int_{K} x^{-1} d x=\int_{0}^{1}\left[(\exp 2 \pi i t) /\left(e_{j}+r_{j} \exp 2 \pi i t\right)\right] 2 \pi i r_{j} d t=z^{\prime}, \text { say. }
$$

If $M$ is a maximal ideal, then $z^{\prime}(M)=\int_{0}^{1}\left(2 \pi i r_{j} \exp 2 \pi i t\right) /\left(e_{j}(M)+r_{j} \exp 2 \pi i t\right) d t$. If $e_{j}(M)=1$, then $z^{\prime}(M)=0$. If $e_{j}(M)=0$, then $z^{\prime}(M)=2 \pi i$. Thus $z^{\prime}(M)$ $=2 \pi i\left(e-e_{j}\right)(M)$ for all $M$. If the radical of $B$ is $\{0\}$, then $z^{\prime}=2 \pi i\left(e-e_{j}\right)$.

Now let us consider the polynomial $p(x)=a_{2} x^{2}+a_{1} x+a_{0}$ where $a_{2}$ is in $G$. We may assume $a_{2}=e$. If $p(x)$ has a zero $z_{1}$, then we may write $p(x)=\left(x-z_{1}\right)$ $\cdot\left(x-z_{2}\right)$. The substitution $y=x-z_{1}$ yields $p(x)=y\left[y-\left(z_{2}-z_{1}\right)\right]=y^{2}-c y$ where $c=z_{2}-z_{1}$. Thus we may confine our attention to polynomials of the form $x^{2}-c x$ when studying polynomials which are of degree 2 and have a zero. We assume $p(x)=x^{2}-c x$ and point out some facts about its zeros.

If $B$ is reducible and $e_{j}$ is an idempotent, then clearly $c e_{j}$ is also a zero. Thus $p(x)$ may have infinitely many zeros. Reducibility is not a necessary condition for $p(x)$ to have more than two zeros. In the algebra $C[0,1]$, if $c=\sin 2 \pi t$, then the polynomial $x^{2}-c x$ has the following zeros besides 0 and $c$ :

the function $r_{1}(t)$ defined by: $\sin 2 \pi t$ for $0 \leqq t \leqq 1 / 2$, and 0 for $1 / 2 \leqq t \leqq 1$;

the function $r_{2}(t)$ defined by: $\sin 2 \pi t$ for $1 / 2 \leqq t \leqq 1$ and 0 for $0 \leqq t \leqq 1 / 2$.

Thus the polynomial has four zeros in $C[0,1]$ although this algebra is irreducible. Further, if we let $c=t \sin (1 / t)$, then $x^{2}-c x$ has infinitely many zeros in $C[0,1]$.

If $B$ is an irreducible algebra with radical $\{0\}$ and $c$ is a regular element in $B$, then 0 and $c$ are the only zeros of $x^{2}-c x$. For if $z$ is such that $z(z-c)=0$, then for every maximal ideal $M$ we have $z(M)[z(M)-c(M)]=0$. Thus $z(M)=0$ or $Z(M)=c(M)$. If $z(M) \neq 0$ for any $M$, then $z=c$. If $z\left(M^{\prime}\right)=0$ for some $M^{\prime}$ in $\mathfrak{M}$, then $z=0$, for if not, by virtue of the connectedness of the spectrum of $z$ there is a maximal ideal $M^{\prime \prime}$ such that $0<z\left(M^{\prime \prime}\right)<\mu$, where $\mu=\min \{|c(M)| M$ in $\mathfrak{M}\}>0$. Then we have $z\left(M^{\prime \prime}\right)\left[z\left(M^{\prime \prime}\right)-c\left(M^{\prime \prime}\right)\right] \neq 0$, which contradicts the assumption that $z$ is a zero of $p(x)$.

Having observed the various modes of behavior of $p(x)$, we proceed to study the rational function $[p(x)]^{-1}$. As above, let $D(q)$ be the set of elements of the form $c+g$ where $g$ varies over $G$. $[p(x)]^{-1}$ is defined for all $x$ in $G \cap D(q)$. We denote this set by $D(p)$. As the intersection of two open sets, $D(p)$ is also open. We shall show that it is not empty. (Remark: It is clear that $D(p)$ is independent of its representation as the intersection of the domains of the factors of $p(x)$; i.e. if $p(x)=(x-z)(x-c+z)$ is any other factorization, then $D(p)=\{z+g \mid g$ in $G\} \cap\{(c-z)+g \mid g$ in $G\}$.)

We recall that $G_{1}$ is a subgroup of $G$ and that $g G_{1}$ denotes the coset of 
$G / G_{1}$ which contains $g$ and that this is also the component of $G$ which contains $g$. Let $\left(c+g G_{1}\right)$ denote the set $\left\{c+g g_{1} \mid g_{1} \in G\right\}$. We call this set the "c-translate" of the component $g G_{1}$. Observe that $\lambda g$ is in $g G_{1}$ for all complex $\lambda$. If $x$ is such that $\|x-\lambda g\|<|\lambda| /\left\|g^{-1}\right\|$ for a given $g$, then $x$ is in $g G_{1}$. Thus, if we choose $\lambda$ so that $\|c\|<|\lambda| /\left\|g^{-1}\right\|$, then $c+\lambda g$ is in $g G_{1} \cap\left(c+g G_{1}\right)$; i.e. for all $g$ in $G$ and all $c$ in $B$ the set $g G_{1} \cap\left(c+g G_{1}\right)$ is not empty, or each component of $G$ intersects its $c$-translate.

A component of $G$ may also intersect the $c$-translate of another component. For example, if $g$ is in $G$ but not in $G_{1}$, and $g_{1}$ is in $G_{1}$, then $\left(c+G_{1}\right)$ $\cap g G_{1}$ contains the element $g$ if we let $c=g-g_{1}$. Thus we have $G \cap(c+G)$ $=\bigcup_{o, h \in G}\left[g G_{1} \cap\left(c+h G_{1}\right)\right]$.

Now, $G \cap(c+G)$ is a union of maximal open connected sets, its components. For arbitrary elements $c$ in an arbitrary algebra $B$, not much is known about the structure of these components. We have seen that each $g G_{1}$ intersects its $c$-translate. It is not known whether this intersection is connected. If it is connected, then clearly it is a component of $D(p)$. In each component of $D(p)$, the formula $[p(x)]^{-1}$ defines a single-valued complete analytic function. We shall investigate its singularities. We write $f(x)$ $=[p(x)]^{-1}$.

If $z$ is a plane singularity of $f(x)$, then $z$ is in $\sim G \cup \sim(c+G)$, where " $\sim$ " denotes the complement of a set. If $z$ is in $\sim G$, then its spectrum contains 0 as an isolated point, and all complex numbers in a neighborhood of 0 are not in the spectrum of $z-c$, with the possible exception of 0 . This condition is sufficient for $z$ to be a plane singularity of $f(x)$. If $z$ is not in $G$ and the algebra is irreducible and has radical $\{0\}$, then $z=0$ since the only element which has a spectrum containing 0 as an isolated point is the zero element. In this case, 0 is not in the spectrum of $z-c=-c$ unless $c=0$. If $c \neq 0$, then $c$ is in $G$. Similarly, if $z$ is not in $(c+G)$, then $z-c=0$. If $c$ is not 0 , then the spectrum of $z$ cannot contain 0 since it is connected. Thus $z=c$ is in $G$ again. This shows that 0 and $c$ are the only possible plane singularities of $f(x)$ when the algebra is irreducible and has radical 0 . They are plane singularities only if $c$ is in $G$. This last condition is also sufficient as the next lemma shows.

LeMma 9.1. If $c$ is in $G$, then $c$ and 0 are simple poles of $f(x)$.

Proof. $f(x)$ is clearly defined for all elements $c+\lambda e$ and $\lambda e$ such that $0<|\lambda|<r$, for some real $r$. The Laurent expansions are

$$
\begin{aligned}
f(x) & =[x(x-c)]^{-1}=(x-c)^{-1}[(x-c)+c]^{-1} \\
& =(x-c)^{-1}\left[e+(x-c) c^{-1}\right]^{-1} c^{-1}=(x-c)^{-1} c^{-1}\left[e-(x-c) c^{-1}+\cdots\right], \\
f(x) & =c^{-1}(x-c)^{-1}-c^{-2}+c^{-3}(x-c)-c^{-4}(x-c)^{2}+\cdots
\end{aligned}
$$

where the series converges super-absolutely at least for $\|x-c\|<1 /\left\|c^{-1}\right\|$; similarly, $f(x)=x^{-1}\left(-c^{-1}\right)\left(e-x c^{-1}\right)^{-1}=-c^{-1} x^{-1}\left[e+x c^{-1}+x^{2} c^{-2}+\cdots\right]$, where this series converges super-absolutely at least for $0<\|x\|<1 /\left\|c^{-1}\right\|$. 
For reducible algebras we have the following

LEMмA 9.2. If $B$ is a reducible algebra with radical $\{0\}$, any plane singularity is a pole of order at most 2.

Proof. Let $z$ be a plane singularity of $f(x)$. The coefficients of the Laurent expansion around $z$ are given by $a_{n}=(1 / 2 \pi i) \int_{K}(x-z)^{-n-1} f(x) d x$ where $K$ is the curve $\{z+(r \exp 2 \pi i t) e\}$ and $r>0$ is such that both $z$ and $z-c$ have their spectra outside the circle $C=\{r \exp 2 \pi i t\}$ except for the point 0 . Then we have

$$
a_{n}=(1 / 2 \pi i) \int_{C}(z+\zeta e)^{-1}(z-c+\zeta e)^{-1}(\zeta e)^{-n-1} d \zeta \text { where } \zeta \text { is on } C,
$$

$a_{n}(M)=(1 / 2 \pi i) \int_{C}(z(M)+\zeta)^{-1}[\zeta+z(M)-c(M)]^{-1} \zeta^{-n-1} d \zeta$ for all $n$ and all $M$ in $\mathfrak{M}$. In particular, for $n=-3, \quad a_{-3}(M)=(1 / 2 \pi i) \int_{C}(z(M)+\zeta)^{-1}[z(M)$ $-c(M)+\zeta]^{-1} \zeta^{2} d \zeta$. There are four cases:

(i) $c(M)=0$ and $z(M) \neq 0$. In this case, $a_{-3}(M)=(1 / 2 \pi i) \int c \zeta^{2}(\zeta+z(M))^{-2} d \zeta$ and since $z(M)$ is exterior to $C, a_{-3}(M)=0$ for all $M$.

(ii) $c(M) \neq 0$ and $z(M)=0$. Then $a_{-3}(M)=(1 / 2 \pi i) \int_{c} \zeta^{-1}(\zeta-c(M))^{-1} \zeta^{2} d \zeta$ $=(1 / 2 \pi i) \int_{C}\left(\zeta^{2} / c(M)\right)[1 /(\zeta-c(M))-1 / \zeta] d \zeta=0$ since $c(M)$ is outside $C$.

(iii) $c(M)=0$ and $z(M)=0$. Then $a_{-3}(M)=(1 / 2 \pi i) \int_{c} d \zeta=0$.

(iv) $c(M) \neq 0$ and $z(M) \neq 0$. Then

$$
\begin{aligned}
a_{-3}(M)= & \frac{1}{2 \pi i} \int_{C} \frac{\zeta^{2}}{-c(M)}\left[\frac{1}{\zeta+z(M)}-\frac{1}{\zeta+z(M)-c(M)}\right] d \zeta \\
= & -\frac{1}{2 \pi i} \int_{C} \frac{1}{c(M)}\left[\frac{\zeta^{2}}{\zeta+z(M)}\right] d \zeta \\
& +(1 / 2 \pi i c(M)) \int_{c} \frac{\zeta^{2} d \zeta}{\zeta+z(M)-c(M)}=0
\end{aligned}
$$

since $z(M)$ and $(z-c)(M)$ lie outside $C$.

Similar treatment may be accorded the coefficients $a_{n}$ for $n<-3$. Since the radical is $\{0\}, a_{n}=0$ for $n \leqq-3$ so that $z$ is a pole of order at most 2 .

In reducible algebras, if $c$ is a pole and $e_{j}$ is an idempotent, then $e_{j} c$ is also a pole since $\left(e_{j} c\right)(M)=e_{j}(M) c(M)$ and $e_{j}(M)=0$ or 1 ; i.e. $\left(e_{j} c\right)(M)=0$ or $c(M)$, and $\left(e_{j} c-c\right)(M)=0$ or $-c(M)$. If $c$ is a pole, then $c(M)=0$ or $|c(M)|$ $>\epsilon>0$. Hence $e_{j} c$ has the requisite spectral properties.

Of course, $c$ may not be a singularity $\left({ }^{2}\right)$ at all; i.e. if $c$ is not in $G$. In reducible algebras, this does not preclude the existence of other singularities as it does in the irreducible case. For example, in the algebra $(m)$, let $c$ be a sequence $\{1 / n\}$, which is not in $G$. Then $z=(1,0,1,1, \cdots, 1, \cdots)$ is a pole of $f(x)$, for the spectrum of $z$ consists of 0 and 1 , while the spectrum of $z-c=\{0,-1 / 2,1-1 / 3, \cdots, 1-1 / n, \cdots\}$ consists of all the numbers in

(2) Hereinafter singularity will mean plane-singularity. 
that sequence and the number 1 . Hence 0 is an isolated point. This is sufficient to make $z$ a plane singularity. The lemma shows that it is a pole.

The above example reveals that a zero of $p(x)$ need not be a singularity of $f(x)$. Conversely, the pole $z$ need not be a zero as the example shows. Thus any attempt to locate zeros by contour integration appears doomed to failure.

We have shown that the residues of $x^{-1}$ can be obtained by integrating on curves of the form $\left\{z_{0}+\phi(t) \cdot e\right\}$ where $z_{0}$ is a pole of $x^{-1}$. This is a type of residue theorem. We should like to prove a similar result for $f(x)$ $=x^{-1}(x-c)^{-1}$.

Let $e_{j}$ be an idempotent and let $\mathfrak{M}_{0 j}=\left\{M \mid e_{j}(M)=0\right\}$ and $\mathfrak{M}_{1 j}$ $=\left\{M \mid e_{j}(M)=1\right\}$. Then $\mathfrak{M}=\mathfrak{M}_{0 j} \cup \mathfrak{M}_{1 j}$ and both sets $\mathfrak{M}_{0 j}$ and $\mathfrak{M}_{1 j}$ are closed in $\mathfrak{M}$, and therefore compact. We have the following

Lemma 9.3. A necessary and sufficient condition for $x c=e_{j}$ to have solutions is that $c(M) \neq 0$ for all $M$ in $\mathfrak{M}_{1 j}$.

Proof. Necessity. If $c_{j} c=e_{j}$, then $c_{j}(M) c(M)=e_{j}(M)=1$ for all $M$ in $\mathfrak{M}_{1 j}$. Hence $c(M) \neq 0$ for all such $M$. If $e_{j}=0$, then $\mathfrak{M}_{1 j}$ is empty and the lemma is trivial. If $e_{j}=e$, then $c_{j}=c^{-1}$.

Sufficiency. We shall prove $c_{j}=\left[e_{j} c+\left(e-e_{j}\right)\right]^{-1} e_{j}$ is a solution of $x c=e_{j}$. First, $\left[e_{j} c+\left(e-e_{j}\right)\right](M)=e_{j}(M) \cdot c(M)+1-e_{j}(M)$. For $M$ in $\mathfrak{M}_{1 j}$, this equals $c(M) \neq 0$. For $M$ in $\mathfrak{M}_{0 j}$, it equals 1 . Thus $\left[e_{j} c+\left(e-e_{j}\right)\right]^{-1}$ exists.

Let $d=c_{j} c$. Then $d\left[e_{j} c+\left(e-e_{j}\right)\right]=e_{j} c$. But $e_{j}\left[e_{j} c+\left(e-e_{j}\right)\right]=e_{j} c$. Therefore, $\left(d-e_{j}\right)\left[e_{j} c+\left(e-e_{j}\right)\right]=0$. Since $\left[e_{j} c+\left(e-e_{j}\right)\right]^{-1}$ exists, $d=e_{j}$, which proves the lemma.

We observe that there may be more than one solution of the above equation. In the algebra $(m)$, for example, if $c=(2,0,0, \cdots)$ and $e_{j}=(1,0,0, \cdots)$, then $c=\left(1 / 2, \delta_{2}, \delta_{3}, \cdots\right)$ where $\delta_{n}$ may be chosen arbitrarily for $n \geqq 2$, except that the $\left\{\left|\delta_{n}\right|\right\}$ must be bounded.

We now prove the main theorem on the residues of $f(x)=x^{-1}(x-c)^{-1}$.

THEOREM 9.1. If the radical of $B$ is $\{0\}$, and $d$ is a residue of $f(x)$, then $d$ is of the form $2 \pi i \sum_{j=1}^{p} n_{j} c_{j} e_{j}$ where $e_{j}^{2}=e_{j}, e_{j} e_{k}=0$ for $j \neq k$, and $c_{j} c=e_{j}$ with $|c(M)|>0$ for $M$ in $\bigcup_{j=1}^{p} \mathbb{M}_{1 j}$.

Conversely, if $|c(M)|>0$ for all $M$ in $\cup_{j=1}^{p} \mathfrak{M}_{1 j}$, then there is a curve $K$ in $G \cap(c+G)$ such that $\int_{K} f(x) d x=d$ where $c d=2 \pi i \sum_{j=1}^{p} n_{j} e_{j}$.

Proof. Suppose $\int_{K} f(x) d x=d$. For all $M$, we have $d(M)=\int_{K(M)} d \zeta / \zeta[\zeta$ $-c(M)]$. Thus $d(M)=(1 / c(M)) 2 \pi$ in where $n=0, \pm 1, \pm 2, \cdots$ if $c(M) \neq 0$. If $c(M)=0$, then $d(M)=0$. Hence, $(c d)(M)=2 \pi i n$ where $n$ is a rational integer or zero. This implies $[\exp (c d)](M)=1$ for all $M$. Since the radical is $\{0\}, \exp (c d)=e$. Therefore, $c d=2 \pi i \sum_{j=1}^{p} n_{j} e_{j}$.

Now, the $\mathfrak{M}_{1 j}$ are disjoint in pairs, for suppose $M$ is in $\mathfrak{M}_{1 j} \cap \mathfrak{M}_{1 k}, j \neq k$. Then $0=\left(e_{j} e_{k}\right)(M)=e_{j}(M) e_{k}(M)=1$, which is a contradiction. If $b=2 \pi i$ - $\sum_{j=1}^{p} n_{j} e_{j}$, we see that $b(M)=2 \pi i n_{k} \neq 0$ for $M$ in $\bigcup_{j-1}^{p} \mathfrak{M}_{1 j}, 1 \leqq k \leqq p$. Hence 
$(c d)(M)=b(M) \neq 0$ for $M$ in $\bigcup_{j-1}^{p} \mathbb{M}_{1 j}$ and therefore $d(M) \neq 0, c(M) \neq 0$ for these $M$.

By the preceding lemma, $c_{j}$ exists for $j=1, \cdots, p$. Let $d^{\prime}=2 \pi i \sum_{j=1}^{p} n_{j} c_{j} e_{j}$. If $M$ is not in $\bigcup_{j=1}^{p} \mathbb{M}_{1 j}$, then $d^{\prime}(M)=0$, and $(c d)(M)=b(M)=0$. If $c(M) \neq 0$, then $d(M)=0$. If $c(M)=0$, then $d(M)=0$ by the third line of this proof. Thus $d(M)=0$ for all $M \notin \bigcup_{j=1}^{p} \mathfrak{M}_{1 j}$. If $M$ is in $\mathfrak{M}_{1 j}$ for some $j$, then $d^{\prime}(M)$ $=2 \pi i n_{j} c_{j}(M)=2 \pi i n_{j} / c(M)$. Since $(c d)(M)=2 \pi i n_{j}$ for this $M$, we have $d(M)=2 \pi i n_{j} / c(M)$. Thus $d(M)=d^{\prime}(M)$ for all $M$. Since the radical is $\{0\}, d=d^{\prime}$ as was to be shown.

Now suppose $b=2 \pi i \sum_{j=1}^{p} n_{j} e_{j}$ where $e_{j}^{2}=e_{j}, e_{j} e_{k}=0$ for $j \neq k$ and $|c(M)|>0$ for $M$ in $\bigcup_{j=1}^{p} \mathfrak{M}_{1 j}$. Let $K$ be the curve parametrized as follows: $z(t)$ $=r \exp (2 \pi i t b)+R\left(e-\sum_{j=1}^{p} e_{j}\right)$ where $0<r<\min \left\{|c(M)|, M\right.$ in $\left.\bigcup_{j=1}^{p} \mathfrak{M}_{1 j}\right\}$ and $R>r+\|c\|$. (Since $\bigcup_{j=1}^{p} \mathfrak{M}_{1 j}$ is compact, $\min _{M \in U \mathfrak{M}_{i j}}\{|c(M)|\}>0$.) We shall prove that $z(t)$ is in $G \cap(c+G)$ for $0 \leqq t \leqq 1$.

Let $M$ be in $\bigcup_{j=1}^{p} \mathfrak{M}_{1 j}$, say $M$ in $\mathfrak{M}_{1 k}$. Then $b(M)=2 \pi i n_{k}$ and $[z(t)](M)$ $=r \exp \left(2 \pi i n_{k} t\right)+R(1-1)=r \exp \left(2 \pi i n_{k} t\right) \neq 0$. If $M^{\prime}$ is in $\cap_{j=1}^{p} \mathfrak{M}_{0 j}$, then $e_{j}\left(M^{\prime}\right)=0$ for $j=1, \cdots, p$. Hence $[z(t)]\left(M^{\prime}\right)=r+R>0$. Thus $[z(t)](M) \neq 0$ for all $M$ in $\mathfrak{M}$; i.e. $z(t)$ is in $G$ for $0 \leqq t \leqq 1$.

Similarly, for $M$ in $\bigcup_{1}^{p} \mathfrak{M}_{1 j},|[z(t)-c](M)|=\mid r$ exp $\left(2 \pi i n_{k} t\right)-c(M) \mid$ $\geqq|c(M)|-r \geqq \mu-r>0$ where $\mu=\min \left\{|c(M)|, M\right.$ in $\left.\cup_{\mathfrak{M}_{1 j}}\right\}$. For $M$ not in $\cup_{\mathfrak{M}_{1 j}}, \quad|[z(t)-c](M)|=|r+R-c(M)| \geqq R-r-|c(M)| \geqq R-r-\|c\|>0$. Thus $[z(t)-c]$ is in $G$ for $0 \leqq t \leqq 1$.

The integrand $f(x)$ is therefore defined for all $x$ on $K$. The integration proceeds as follows:

$$
\begin{aligned}
& \int_{K} f(x) d x \\
& =\int_{0}^{1} \frac{2 \pi i b r \exp (2 \pi i t b) d t}{\left[r \exp (2 \pi i t b)+R\left(e-\sum_{1}^{p} e_{j}\right)\right]\left[r \exp (2 \pi i t b)+R\left(e-\sum_{1}^{p} e_{j}\right)-c\right]} \\
& =\sum_{j=1}^{p} e_{i} \int_{0}^{1} \frac{2 \pi i b r \exp (2 \pi i t b) d t}{z(t)[z(t)-c]}=\sum_{j=1}^{p} e_{j} \int_{K}^{x^{-1}(x-c)^{-1} d x} \\
& =\sum_{j=1}^{p} \int_{K} e_{j} x^{-1}(x-c)^{-1} d x=\sum_{j=1}^{p} \int_{K} c_{j}\left[(x-c)^{-1}-x^{-1}\right] d x
\end{aligned}
$$

since $c_{j} c=e_{j}$ and $c_{j}$ exists by the Lemma 9.3.

Now let $I_{0}=\int_{K} x^{-1} d x=\int_{0}^{1}[2 \pi i b r \exp (2 \pi i t b)] \cdot[z(t)]^{-1} d t$. For $M$ in $\bigcup_{j-1}^{p} \mathbb{R}_{1 j}$, $I_{0}(M)=\int_{0}^{1} 2 \pi i n_{k} r \exp \left(2 \pi i n_{k} t\right) /\left(r \exp 2 \pi i n_{k} t\right) d t=2 \pi i n_{k}$. For $M$ not in UM $\mathbb{M}_{1 j}$, $I_{0}(M)=\int_{0}^{1} 0 /(r+R) d t=0$. Thus $I_{0}(M)=b(M)$ for all $M$. Since the radical is $\{0\}, I_{0}=b$.

Let $I_{c}=\int_{K}(x-c)^{-1} d x=\int_{0}^{1} 2 \pi i b r \exp (2 \pi i t b)[z(t)-c]^{-1} d t$. For $M$ in $\bigcup_{j=1}^{p} \mathfrak{M}_{1 j}$, 


$$
I_{c}(M)=\int_{0}^{1} \frac{2 \pi i n_{k} r \exp \left(2 \pi i n_{k} t\right)}{r \exp \left(2 \pi i n_{k} t\right)-c(M)} d t .
$$

Letting $\zeta(t)=r \exp \left(2 \pi i n_{k} t\right)$, we have $d \zeta=2 \pi i n_{k} \zeta d t$ so that

$$
I_{c}(M)=\int_{K^{\prime}}\left(\frac{1}{\zeta-c(M)}\right) d \zeta
$$

where $K^{\prime}$ is the closed curve $r \exp \left(2 \pi i n_{k} t\right), 0 \leqq t \leqq 1$, in the complex plane. Since $|c(M)| \geqq \mu>r, c(M)$ lies outside of $K^{\prime}$ for all $M$ in $\bigcup_{1}^{p} \mathfrak{M}_{1 j}$. Hence, $I_{c}(M)=0$. Likewise, for $M$ not in $\bigcup_{1}^{p} \mathfrak{M}_{1 j}, I_{c}(M)=\int_{0}^{1} 0 /(R+r-c(M)) d t$ which equals 0 . Thus $I_{c}=0$.

Therefore, $\int_{K} f(x) d x=-\sum_{j=1}^{p} c_{j} b$. Since $b=2 \pi i \sum_{j=1}^{p} n_{j} e_{j}$, and $c_{j} e_{k}=0$ for $j \neq k$ (i.e. $\left[c_{j} e_{k}\right](M)=c_{j}(M) e_{k}(M)=0$ since $c_{j}(M)=0$ for $M$ in $\mathfrak{M}_{1 k}$ and $e_{k}(M)=0$ for $M$ not in $\mathfrak{M}_{1 k}$ ), we have

$$
\int_{K} f(x) d x=-2 \pi i \sum_{j=1}^{p} n_{j} c_{j} e_{j} .
$$

Reversing the parametrization of $K$ then yields the desired result.

We now demonstrate that all residues of $f(x)$ may be expressed as a sum of residues obtained by integrating on curves of the form $\left\{z_{0}+\psi(t) e\right\}$ where $z_{0}$ is a pole of $f(x)$ and $\psi(t)$ is a continuous complex function, $0 \leqq t \leqq 1$. This is an analogue of the residues theorem for $f(x)$.

Let $d$ be a residue of $f(x)$. Thus $d=2 \pi i \sum_{j=1}^{p} n_{j} c_{j} e_{j}$. Again, let $\mu$ $=\min \left\{|c(M)|, M\right.$ in $\left.U \mathfrak{M}_{1 j}\right\}$ and $\mu>r>0, R>r+\|c\|$.

Theorem 9.2. The elements $z_{j}=R\left(e-\epsilon_{j}\right), j=1, \cdots, p$, are poles of $f(x)$ and the integral of $f(x)$ on the curve $\left.K_{j}:\left\{z_{j}+r \exp 2 \pi i n_{j} t\right), 0 \leqq t \leqq 1\right\}$ is equal to $-2 \pi i n_{j} c_{j}$. Thus $\sum_{j-1}^{p} \int_{K_{j}} f(x) d x=-d$.

To prove that $z_{j}$ is a pole we must show that $f\left(z_{j}+\lambda e\right)$ is defined for $0<|\lambda|<r+\epsilon$, where $r+\epsilon\|c\|<R, \epsilon>0, r+\epsilon<\mu$.

If $M$ is in $\mathfrak{M}_{1 j}$, then $\left|\left(z_{j}+\lambda e\right)(M)\right|=|0+\lambda| \neq 0$. If $M$ is not in $\mathfrak{M}_{1 j}$, $\left|\left(z_{j}+\lambda e\right)(M)\right|=|R+\lambda| \geqq R-|\lambda|>0$. Thus $\left(z_{j}+\lambda e\right)$ is in $G$ for all $\lambda$ such that $0<|\lambda|<r+\epsilon$. Likewise, $\left|\left(z_{j}+\lambda e-c\right)(M)\right|=|\lambda-c(M)| \geqq|c(M)|-|\lambda|$ $\geqq \mu-(r+\epsilon)>0$ for $M$ in $\mathfrak{M}_{1 j}$ and $\left|\left(z_{j}+\lambda e-c\right)(M)\right|=|R+\lambda-c(M)| \geqq R$ - $-\|c\|-r-\epsilon>0$ for $M$ not in $\mathfrak{M}_{1 j}$. Thus $\left(z_{j}+\lambda e\right)$ is in $(c+G)$ and $z_{j}$ is a pole.

Let $d_{j}=\int_{K_{j}} f(x) d x=\int_{0}^{1} 2 \pi i n_{j} r \quad \exp \quad\left(2 \pi i n_{j} t\right)\left[z_{j}+r \quad \exp 2 \pi i n_{j} t\right]^{-1}\left[z_{j}+r\right.$ $\left.\cdot \exp 2 \pi i n_{j} t-c\right]^{-1} d t$. Then

$$
d_{j}(M)=\int_{0}^{1} \frac{2 \pi i n_{j} r \exp \left(2 \pi i n_{j} t\right) d t}{r \exp \left(2 \pi i n_{j} t\right)\left[r \exp \left(2 \pi i n_{j} t\right)-c(M)\right]}=\int_{K_{j}(M)} \frac{d \zeta}{\zeta[\zeta-c(M)]}
$$

for $M$ in $\mathfrak{M}_{1 j}$. Here $K_{j}(M)=\left\{r \exp \left(2 \pi i n_{j} t\right)\right\}$. Since $|c(M)|>r$ for $M$ in $\mathfrak{M}_{1 j}, d_{j}(M)=-2 \pi i n_{j} / c(M)$. For $M$ in $\mathfrak{M}_{0 j}$, we have 


$$
\begin{aligned}
d_{j}(M) & =\int_{0}^{1} \frac{2 \pi i n_{j} r \exp \left(2 \pi i n_{j} t\right) d t}{\left[R+r \exp 2 \pi i n_{j} t\right]\left[R+r \exp \left(2 \pi i n_{j} t\right)-c(M)\right]} \\
& =\int_{K_{j}(M)} \frac{d \zeta}{\zeta[\zeta-c(M)]}=0
\end{aligned}
$$

since $\zeta=R+r \exp \left(2 \pi i n_{j} t\right)$ and $|\zeta| \geqq R-r>\|c\| \geqq|c(M)|$; i.e. $c(M)$ is exterior to $K_{j}(M)=\left\{R+r \exp \left(2 \pi i n_{j} t\right)\right\}$. Thus $d_{j}(M) c(M)=-2 \pi i n_{j} e_{j}(M)$ for all $M$. Since the radical is $\{0\}, d_{j} c=-2 \pi i n_{j} e_{j}$, or $d_{j}=-2 \pi i n_{j} c_{j}$. Clearly, $\sum_{j=1}^{p} d_{j}$ $=-d$ or $\sum_{j=1}^{p} \int_{K j} x^{-1}(x-c)^{-1} d x=-d$ as was to be shown.

We conclude the discussion of the function $x^{-1}(x-c)^{-1}$ with some further remarks on the topological structure of its domain $G \cap(c+G)$.

If $c$ is in $G$, then the component $c G_{1}$ of $G$ intersects every component of $(c+G)$. In proof, let $\left(c+g G_{1}\right)$ be any component of $(c+G)$. Let $g_{1}$ be an element of $G_{1}$ such that $\left\|g_{1}\right\|\|g\|<1 /\left\|c^{-1}\right\|$. Then we have $\left\|\left(c+g g_{1}\right)-c\right\|$ $=\left\|g g_{1}\right\| \leqq\|g\|\left\|g_{1}\right\|<1 /\left\|c^{-1}\right\|$ and $\left(c+g g_{1}\right)$ is in $c G_{1}$. Correspondingly, the component $\left(c+c G_{1}\right)$ intersects every component of $G$, for if $g$ in $G$ is of sufficiently small norm, then $g-c$ is in $c G_{1}$ and $c+(g-c)=g$ is in $\left(c+c G_{1}\right)$.

This by no means determines the set-theoretic structure of $G \cap(c+G)$ for it is not known whether a component $g G_{1}$, other than $c G_{1}$, intersects any component of $(c+G)$ except its translate $\left(c+g G_{1}\right)$ and $\left(c+c G_{1}\right)$, or whether these intersections are connected.

The discussion for $f(x)=x^{-1}(x-c)^{-1}$ may be extended to the case $\left[p_{n}(x)\right]^{-1}$ where $p_{n}(x)$ is a polynomial which is of degree $n$ and splits into $n$ linear factors. If $p_{n}(x)$ does not split into linear factors, the situation is more difficult. If $D\left(p_{n}\right)$ denotes the set of $z$ in $B$ such that $\left[p_{n}(z)\right]^{-1}$ exists and $O\left(p_{n}\right)$ is its complement, then we can state the following

TheOREM 9.3. If $p_{n}(x)=a_{0}+a_{1} x+\cdots+a_{n} x^{n}$ is such that $O\left(p_{n}\right)$ and $D\left(p_{n}\right)$ are not empty, then $f_{n}(x)=\left[p_{n}(x)\right]^{-1}$ is a meromorphic function; i.e. it has no plane singularities other than poles. In fact, the poles are of order not greater than $n$, assuming that the radical is $\{0\}$.

Proof. The domain of analyticity of $f_{n}(x)$ consists of the various components of $D\left(p_{n}\right)$. Suppose $z_{0}$ is a plane singularity. Then $z_{0}$ is in $O\left(p_{n}\right)$ and $z_{0}+\lambda e$ is in $D\left(p_{n}\right)$ for all $\lambda$ such that $0<|\lambda|<r_{1}$. Let $f_{n}(x)=\sum_{-\infty}^{\infty} b_{m}\left(x-z_{0}\right)^{m}$ be the Laurent expansion around $z_{0}$, where $b_{m}=(1 / 2 \pi i) \int_{K} f_{n}(x)\left(x-z_{0}\right)^{-m-1} d x$, $m=0, \pm 1, \pm 2, \cdots$, and $K$ is the curve $\left\{z_{0}+(r \exp 2 \pi i t) e\right\}, 0<r<r_{1}$. For $M$ in $\mathfrak{M}$, we have

$b_{m}(M)=\frac{1}{2 \pi i} \int_{K(M)} \frac{\lambda_{t}^{-m-1} d \lambda_{t}}{\left(a_{0}(M)+a_{1}(M) \zeta+\cdots+a_{N}(M) \zeta^{N}\right)}$ where $a_{N}(M) \neq 0$ and $0<N \leqq n$ and $\zeta=z_{0}(M)+\lambda_{t}, \lambda_{t}=r \exp (2 \pi i t)$. Factoring the complex poly- 
nomial, we obtain $a_{0}(M)+a_{1}(M) \zeta+\cdots+a_{N}(M) \zeta^{N}=\left[a^{N}(M)\right]\left(\zeta-\lambda_{1}\right) \cdots$ $\left(\zeta-\lambda_{N}\right)$. Thus

$$
b_{m}(M)=\frac{1}{2 \pi i} \int_{K(M)} \frac{\lambda_{t}^{-m-1} d \lambda_{t}}{a_{N}(M)\left[\lambda_{t}-\left(\lambda_{1}-z_{0}(M)\right)\right] \cdots\left[\lambda_{t}-\left(\lambda_{N}-z_{0}(M)\right)\right]} .
$$

If $\lambda_{k}-z_{0}(M) \neq 0$, then $\left|\lambda_{k}-z_{0}(M)\right| \geqq r_{1}$ for $k=1, \cdots, N$, for otherwise $z_{0}+\left(\lambda_{k}-z_{0}(M)\right) e$ is in $D\left(p_{n}\right)$. This is impossible because

$$
\begin{gathered}
{\left[p_{n}\left(z_{0}+\left(\lambda_{k}-z_{0}(M) e\right)\right](M)=a_{N}(M)\left[z_{0}(M)+\lambda_{k}-z_{0}(M)-\lambda_{1}\right]\right.} \\
\cdots\left[z_{0}(M)+\lambda_{k}-z_{0}(M)-\lambda_{N}\right]
\end{gathered}
$$

which equals zero. Thus for $m<-n$, the integrand in the expression for $b_{m}(M)$ is analytic interior to the curve $\lambda_{t}=r \exp (2 \pi i t)$ and also on the curve. Hence, for such $m, b_{m}(M)=0$. This holds for all $M$. Since the radical is $\{0\}, b_{m}=0$.

As a concluding remark, we observe that the theory of the general rational function $p_{m}(x)\left[p_{n}(x)\right]^{-1}$, where $p_{n}(x)$ and $p_{m}(x)$ are polynomials, is not immediately obtainable from the theory of $\left[p_{n}(x)\right]^{-1}$ because the domain of analyticity may possibly extend beyond $D\left(p_{n}\right)$.

\section{BIBLIOGRAPHY}

1. E. R. Lorch, The theory of analytic functions in normed abelian vector rings, Trans. Amer Math. Soc. vol. 54 (1943) pp. 414-425.

2. - The structure of normed abelian rings, Bull. Amer. Math. Soc. vol. 50 (1944) pp. $447-463$.

3. I. Gelfand, Normierte Ringe, Rec. Math. (Mat. Sbornik) N.S. vol. 9 (51) (1941) pp. 3-24.

4. E. Hille, Functional analysis and semi-groups, Amer. Math. Soc. Colloquium Publications, vol. $31,1948$.

5. N. Dunford, Spectral theory, Bull. Amer. Math. Soc. vol. 48 (1943) pp. 637-651.

6. A. E. Taylor, Analysis in complex Banach spaces, Bull. Amer. Math. Soc. vol. 49 (1943) pp. $652-669$.

7. M. Nagumo, Einige analytische Untersuchungen in linearen metrischen Ringen, Jap. J. Math. (1936).

8. S. Mazur, Sur les anneaux lineaires, C. R. Acad. Sci. Paris vol. 207 (1938) p. 1025.

9. I. Gelfand and G. Silov, Über verschiedene Methoden der Einführung der Topologie in die Menge der maximalen Ideale eine normierten Ringes, Rec. Math. (Mat. Sbornik) N.S. vol. 9 (51) (1941) pp. 25-39.

10. C. E. Rickart, B-algebras with an adjoint operation, Ann. of Math. vol. 47 (1946) pp. 528-550.

11. - Singular elements of a B-algebra, Duke Math. J. vol. 14 (1947) pp. 1063-1077.

12. E. K. Blum, The fundamental group of the principal component of a commutative Banach algebra, Proc. Amer. Math. Soc. vol. 4 (1953) pp. 397-400.

Columbia University,

NEW YORK, N. Y.

UNIVERSITY OF MARYLAND,

College Park, MD. 